\title{
ARTICLE
}

\section{7q21 asthma-risk variants switch CTCF binding and regulate IL-2 production by T cells}

Benjamin Joachim Schmiedel ${ }^{1}$, Grégory Seumois ${ }^{1}$, Daniela Samaniego-Castruita', Justin Cayford ${ }^{1}$, Veronique Schulten ${ }^{1}$, Lukas Chavez ${ }^{2}$, Ferhat $A y^{1}$, Alessandro Sette ${ }^{1}$, Bjoern Peters ${ }^{1} \&$ Pandurangan Vijayanand ${ }^{1,3}$

Asthma and autoimmune disease susceptibility has been strongly linked to genetic variants in the 17q21 haploblock that alter the expression of ORMDL3; however, the molecular mechanisms by which these variants perturb gene expression and the cell types in which this effect is most prominent are unclear. We found several 17q21 variants overlapped enhancers present mainly in primary immune cell types. $\mathrm{CD} 4{ }^{+} \mathrm{T}$ cells showed the greatest increase (threefold) in ORMDL3 expression in individuals carrying the asthma-risk alleles, where ORMDL3 negatively regulated interleukin-2 production. The asthma-risk variants rs 4065275 and rs12936231 switched CTCF-binding sites in the 17q21 locus, and 4C-Seq assays showed that several distal cis-regulatory elements upstream of the disrupted ZPBP2 CTCF-binding site interacted with the ORMDL3 promoter region in $\mathrm{CD}^{+}{ }^{+} \mathrm{T}$ cells exclusively from subjects carrying asthma-risk alleles. Overall, our results suggested that $\mathrm{T}$ cells are one of the most prominent cell types affected by $17 q 21$ variants.

\footnotetext{
${ }^{1}$ La Jolla Institute for Allergy and Immunology, La Jolla, California 92037, USA. ${ }^{2}$ German Cancer Research Center (DKFZ), Division of Pediatric Neurooncology, 69120 Heidelberg, Germany. ${ }^{3}$ Clinical and Experimental Sciences, National Institute for Health Research Southampton Respiratory Biomedical Research Unit, University of Southampton, Faculty of Medicine, Southampton SO16 6YD, UK. Correspondence and requests for materials should be addressed to P.V. (email: vijay@lji.org).
} 
A sthma, allergy and autoimmune diseases such as diabetes, Crohn's disease, ulcerative colitis, psoriasis, rheumatoid arthritis and systemic lupus erythematosus are some of the most common chronic diseases affecting people around the world ${ }^{1-8}$. Strong evidence of heritability from twin studies has prompted large-scale genome-wide association studies (GWAS) to pinpoint the genetic risk factors that drive the pathogenesis of these complex diseases ${ }^{9}$. Several thousand common singlenucleotide polymorphisms (SNPs) associated with disease susceptibility have been identified; however, the vast majority of these SNPs are located in non-coding regions of the genome, and thus it has been challenging to define how these SNPs are related to the disease ${ }^{10}$. Moreover, the cell type(s) where disease riskassociated SNPs have the most prominent effects are unknown, thus hampering functional studies required to successfully translate GWAS discoveries to improvements in disease management. The vast array of immune and structural cell types involved in disease pathogenesis further compound this problem.

Based on recent papers from the ENCODE Project Consortium $^{11,12}$ and our own analyses ${ }^{13}$, there is now overwhelming evidence that many disease-associated genetic variants can perturb the functions of cis-regulatory DNA that controls the expression of one or more neighbouring genes on the same allele, thereby influencing gene expression and disease outcome. In many cases these cis-regulatory regions are cell type-specific ${ }^{11}$, such that a region and its disease-associated genetic variants influence gene expression in just a subset of all possible cell types in the body, and therefore, these susceptible cell types are likely to be major drivers of the genetic risk for that specific disease. Thus, identifying the cell type in which cis-regulatory regions bearing disease-associated SNPs are selectively active may indicate the precise cell types that initiate or maintain disease pathogenesis. For example, by overlapping enhancers of naive and memory $\mathrm{CD} 4{ }^{+} \mathrm{T}$-cell subsets with all known asthma-risk SNPs, we recently showed strong enrichment of these SNPs in enhancers of memory $\mathrm{CD} 4{ }^{+} \mathrm{T}$ cells that produce type 2 cytokines ( $\mathrm{T}_{\mathrm{H}} 2$ cells), implying an important role for $\mathrm{T}_{\mathrm{H}} 2$ cells in asthma pathogenesis ${ }^{14}$.

In this study, we take a similar unbiased approach to determine the cell types that are most susceptible to the effects of SNPs located in the $17 \mathrm{q} 12-\mathrm{q} 21$ genetic risk locus. Originally the $17 \mathrm{q} 21$ locus was strongly linked to asthma susceptibility, which was confirmed by multiple other GWAS studies in diverse ethnic populations ${ }^{15-20}$ and in a large study of individuals with severe asthma $^{21}$. Subsequently, various other autoimmune diseases such as type 1 diabetes, Crohn's disease, ulcerative colitis, psoriasis, rheumatoid arthritis, systemic lupus erythematosus and primary biliary cirrhosis were all found to share the same 17q21 risk locus, suggesting its broader implication in several diseases ${ }^{2-8,22}$; however, an important distinction is that the risk alleles for asthma paradoxically have a protective effect for the autoimmune diseases studied ${ }^{2-6,22}$. Despite the robustness of these associations with asthma and other autoimmune diseases, the molecular mechanisms by which $17 \mathrm{q} 21$ SNPs perturb gene expression and/or predispose carriers to disease are unclear.

The 17q21 locus harbours a dense haploblock of 136 SNPs in tight linkage disequilibrium that overlap six gene loci: IKZF3, ZPBP2, GSDMB, ORMDL3, LRRC $3 C$ and GSDMA $A^{1,23}$, of which the expression of two genes (ORMDL3 and GSDMB) has been shown to be modestly affected by the asthma-risk SNPs in the locus ${ }^{24-26}$. A recent study that linked genotype to phenotype showed that children carrying the $17 \mathrm{q} 21$ asthma-risk SNP (rs7216389) were at higher risk of developing wheeze following rhinovirus infection (common cold), an event that is an independent predictor of asthma development later in $l i f e^{26}$.
Although ORMDL3 expression is elevated in children with asthma as well as in individuals with the $17 \mathrm{q} 21$ riskhaploblock ${ }^{1,23,25,27}$, the biological functions of ORMDL3 that are relevant to asthma pathogenesis are also poorly defined.

Here we systematically examine in which cell types the $17 \mathrm{q} 21$ risk haploblock has the most effect on ORMDL3 expression, how that effect is mediated by disruption of cis-regulatory elements, and how cytokine production is thereby affected in $\mathrm{T}$ cells. We show that ORMDL3 expression is most perturbed by the $17 \mathrm{q} 21$ asthma-risk SNPs in primary T cell subsets and B cells. Assessing $\mathrm{H} 3 \mathrm{~K} 27 \mathrm{ac}$ (chromatin mark of active enhancers) enrichment levels in the $17 \mathrm{q} 21$ locus revealed an active enhancer in the first intron of ORMDL3 that displayed genotype-dependent changes in activity. Further, we discovered that an asthma-risk SNP in this enhancer favours the binding of CTCF (rs4065275), while in contrast another linked SNP downstream of this site prevented CTCF binding (rs12936231), leading to alteration of CTCFbinding patterns in the locus. The three-dimensional (3D) organization of the $17 \mathrm{q} 21$ locus in $\mathrm{CD}^{+} \mathrm{T}$ cells was also modified in the asthma-risk alleles to favour recruitment of distal cis-regulatory elements to the ORMDL3 promoter region. Knocking down transcript levels of ORMDL3 in memory $\mathrm{CD} 4{ }^{+} \mathrm{T}$ cells significantly increased the production of interleukin (IL)-2 following T-cell receptor stimulation.

\section{Results}

17q21 asthma-risk SNPs locus overlap immune cell enhancers. The majority of SNPs $(94 \%, 128 / 136)$ present in the $17 q 21$ asthma-risk haploblock are located in intronic or intergenic noncoding sequences (non-coding SNPs, ncSNPs) whose cell-specific cis-regulatory potential has not been fully characterized (Fig. 1a,b); as a result, the specific cell type that is most affected by the $17 \mathrm{q} 21$ asthma-risk variants is largely unknown. This is especially important for investigating the genetic basis of diseases such as asthma where several immune and structural cells present in the lungs have been implicated in disease pathogenesis ${ }^{28-30}$. Here we utilized the comprehensive DNase I hypersensitivity sites (DHS) data sets, generated by the ENCODE Project ${ }^{31}$ and NIH Epigenomics Roadmap Consortiums ${ }^{32}$, to first define the cell types where the $17 \mathrm{q} 21$ locus is selectively more active, that is, harbour a significantly higher number of cis-regulatory elements (DHS), as these cell types are more likely to be affected by the ncSNPs present in the locus. A total of 462 unique DHS were present in the 17q21 locus (Fig. 1a) and immune cell types had significantly more DHS when compared with non-immune cell types $(P<0.001$ by Student's unpaired two-tailed $t$-test, and following Bonferroni correction for multiple testing, Fig. 1c and see Methods); this enrichment of immune cell DHS was not observed in many of the other asthma-risk haploblocks identified by GWAS studies (Supplementary Fig. 1a and Supplementary Data set 2), suggesting that this effect in immune cells is not generic for all GWAS asthma-risk haploblocks. By ordering 62 primary cell types based on the number of DHS present, we observed that lymphocytes ( $\mathrm{T}$ cell subsets, $\mathrm{B}$ cells and natural killer (NK) cells) ranked in the top 10, harbouring well over 50 DHS (Fig. 1d), whereas monocytes, lung structural cells including bronchial epithelial cells (BECs), fibroblasts and endothelial cells, and other irrelevant tissue types (for example, brain) harboured relatively fewer DHS. Among fetal tissues, the greatest number of DHS was observed in thymus and spleen (organs rich in immune cells) instead of lung tissue (the target organ affected in asthma) (Supplementary Fig. 1b). Altogether, these data indicate that the locus is highly active in several immune cell types, and that several $17 \mathrm{q} 21$ cis-regulatory regions are highly immune cell-specific (Fig. 1a,c,d). 
a

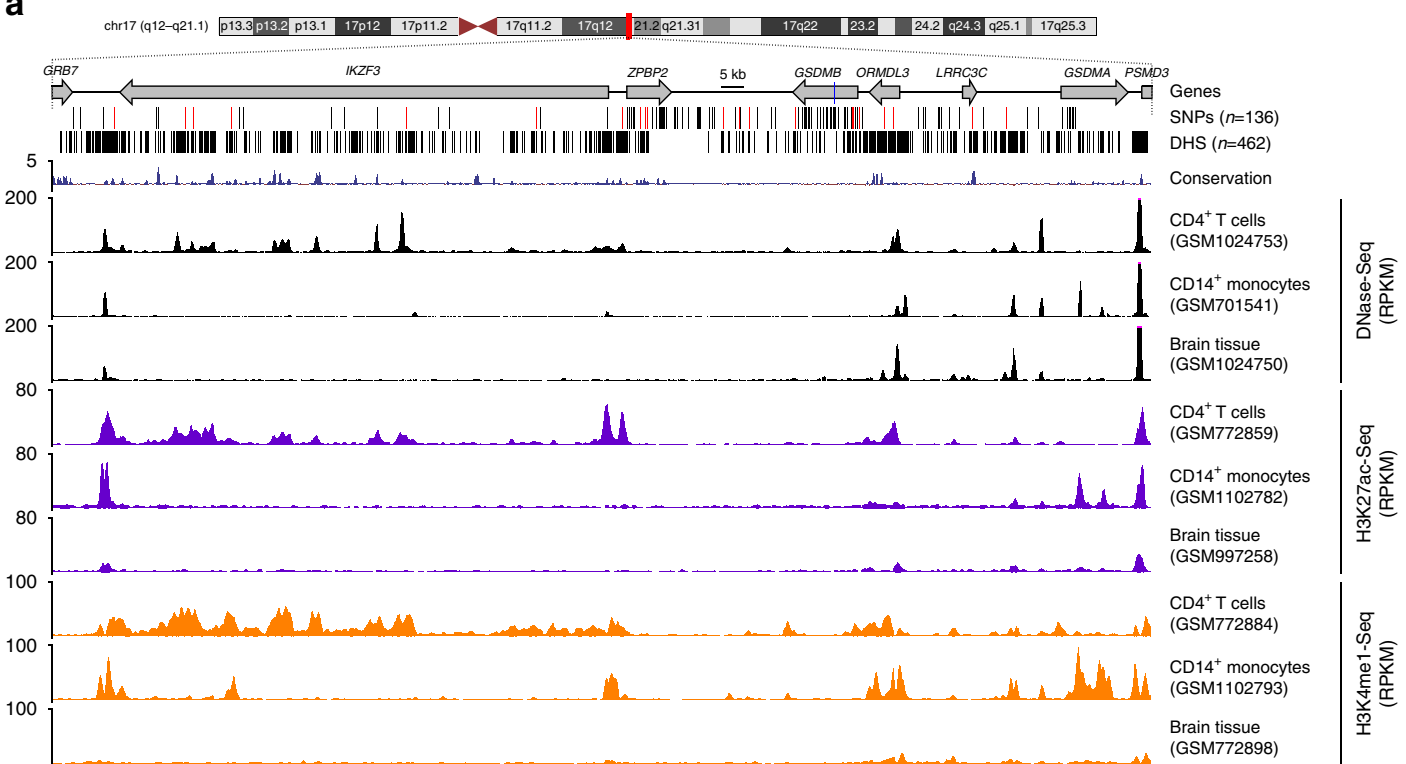

b $17 \mathrm{q} 21 \mathrm{SNPs}$

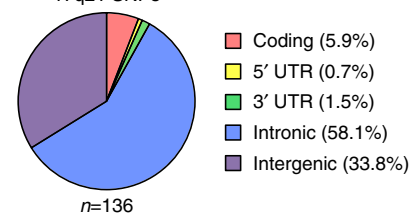

d

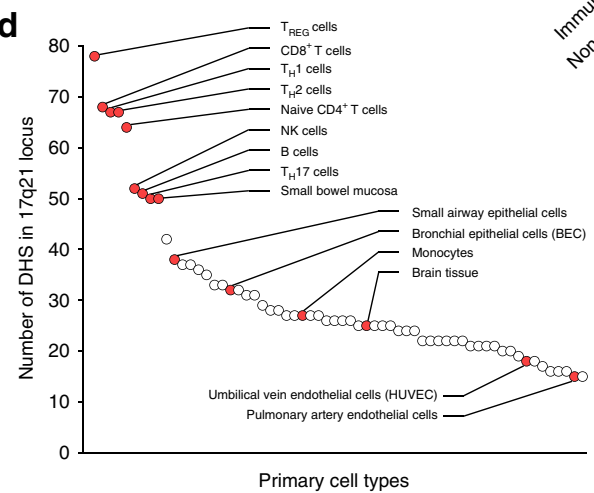

C
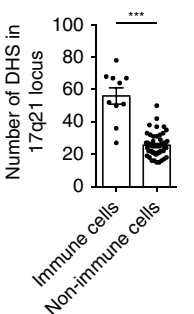

e

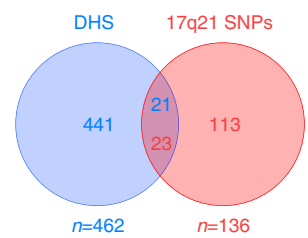

f

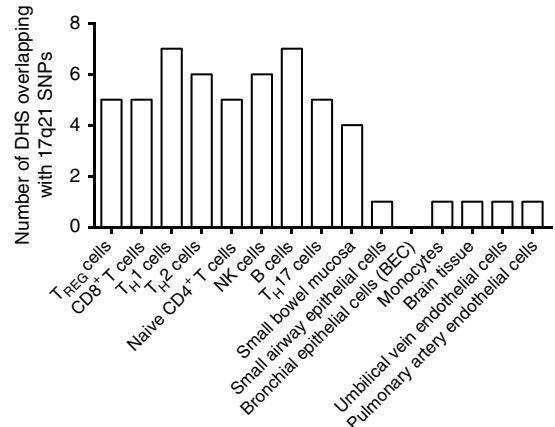

Figure 1 | 17q21 SNPs overlap immune cell enhancers. (a) University of California Santa Cruz (UCSC) tracks showing chromosomal location and genes present in the 17q21 locus, containing a large haplotype block of asthma-associated SNPs; the location of asthma-risk SNP rs7216389 is indicated as blue line in the gene track. Black lines indicate SNPs' genomic location, and red lines are SNPs that overlap peaks of DNase hypersensitivity sites (DHS) from multiple cell types obtained from the ENCODE Encyclopedia (version 2) provided by the ENCODE Project Consortium (see Methods). Exemplary DHS tracks, H3K27ac and H3K4me1 enrichment tracks from CD4 ${ }^{+}$T cells, CD14 ${ }^{+}$monocytes and brain tissue (from ENCODE Project and NIH Epigenomics Roadmap Consortiums) are shown along with UCSC multispecies conservation tracks. (b) Distribution of asthma-associated 17q21 SNPs in different genomic regions. (c) The average number of DHS in the 17q21 locus of immune versus non-immune cell types $(n=10$ and $n=52$, respectively) and (d) the 62 primary cell types (indicated as dots, profiled by the ENCODE Project Consortium, see Methods) ordered based on the number of DHS in the 17q21 locus. The top hits and discussed cell types are named and marked in red. (e) Overlap of DHS and 17q21 SNPs. (f) Number of DHS that directly overlap 17q21 SNPs in various cell types (full list in Supplementary Data set 2b). Error bars are mean \pm s.e.m.; ${ }^{\star \star \star} P<0.001$ by Student's unpaired two-tailed $t$-test, and following Bonferroni correction for multiple testing.

About $17 \%(23 / 136)$ of the SNPs in the $17 \mathrm{q} 21$ asthma-risk haploblock directly overlap DHS in the locus (Fig. 1e). Importantly, more than five of the DHS present in immune cells, such as T-cell subsets, B cells and natural killer cells, directly overlap asthma-risk SNPs, whereas DHS present in monocytes, epithelial cells or other irrelevant tissue (brain) overlap one or no asthma-risk SNPs (Fig. 1f). Thus, our in silico analysis of enhancer profiles suggests that expression of $17 \mathrm{q} 21$ genes is most likely to be perturbed in immune cell types.
ORMDL3 expression is most affected in primary $\mathrm{T}$ cells. In 10 primary immune cell types freshly isolated from peripheral blood of 34 subjects enrolled in the La Jolla Institute for Allergy and Immunology's (LJI) Normal Blood Donor Program (Supplementary Fig. 2), we assessed expression of two genes in the 17q21 locus (ORMDL3 and GSDMB) whose expression levels have been shown to be affected by $17 \mathrm{q} 21$ SNPs in previous studies $^{24-26}$. Compared with non-immune cells, such as BECs, lung cancer cell lines (A549), and human umbilical vein 
endothelial cells, both genes were expressed at relatively high levels in most primary immune cell types with the exception of monocytes and dendritic cells (Fig. 2a). Among the 34 subjects, expression of ORMDL3 and GSDMB genes were both correlated between the cell types of higher transcriptional activity (Spearman correlation value of 0.74 and 0.83 , respectively, between naive $\mathrm{CD}^{+} \mathrm{T}$ cells and $\mathrm{CD} 8{ }^{+} \mathrm{T}$ cells, Fig. $2 \mathrm{~b}$ and Supplementary Fig. 3a). Though the expression levels of $O R M D L 3$ and GSDMB were variable across donors (Fig. 2a,b), the two were positively correlated across the 34 donors (Spearman correlation value of 0.63 in naive $\mathrm{CD} 4{ }^{+} \mathrm{T}$ cells, Fig. 2c), suggesting a potentially co-regulated expression of these two genes in several cell types (Supplementary Fig. 3b).

Based on genotype at rs7216389 (see Supplementary Data set 3$)^{1}$, located in the gene body of GSDMB, subjects were classified into three groups: (i) homozygous for the asthma-risk allele (TT genotype, $n=9$ ), (ii) homozygous for the non-risk allele (CC genotype, $n=9$ ) and (iii) carrying heterozygous alleles (TC genotype, $n=16$ ). Previous studies have reported a modest increase ( $\sim 1.3$ to 1.8 -fold in peripheral blood mononuclear cells (PBMCs) and lymphoblastoid cell lines) $)^{1,24,26,27}$ in the expression of ORMDL3 and GSDMB transcripts in subjects carrying the asthma-risk alleles. In comparison, we found a much greater increase in purified primary immune cell types, with some cell types such as naive CD4 ${ }^{+} \mathrm{T}$ cells and $\mathrm{B}$ cells displaying a nearly threefold increase in ORMDL3 expression (Fig. 2d, left panel and Fig. 2e). In sharp contrast, monocytes and dendritic cells showed no genotype-dependent effect on gene expression (Fig. 2d, right panel), implying that the effect of the $17 \mathrm{q} 21$ asthma-risk variants is cell-specific and restricted to some primary immune cell types. These findings concur with our prediction based on overlap of $17 \mathrm{q} 21$ asthma-risk variants with cell-specific cis-regulatory elements (Fig. 1f).

We noted that the effect of asthma-risk variants on ORMDL3 expression was completely lost when primary naive CD4 ${ }^{+} \mathrm{T}$ cells were expanded in culture for a few days (Fig. 2f), suggesting in vitro expansion per se may dampen the effects of the $17 \mathrm{q} 21$ variants. Recent studies have shown that $17 \mathrm{q} 21$ risk variants have only modest effects on ORMDL3 expression in in vitro expanded human lymphoblastoid cell lines ${ }^{1,2} 7$, and in the case of primary BECs these variants exert no effect ${ }^{33}$. Such divergent effects of the asthma-risk variants among cell types indicate that the upstream pathway positively regulating the ORMDL3 locus is sensitive to the effects of genetic variants only in certain cell types; therefore, the functional consequences of the underlying genetic risk are likely to be best observed in the cell types most sensitive to the effects of asthma-risk variants, in this case $\mathrm{T}$ cell subsets. Since $\mathrm{CD}^{+}{ }^{+} \mathrm{T}$ cells have a central role in orchestrating asthma pathogenesis ${ }^{34}$, we focused our functional studies on this subset of $\mathrm{T}$ cells.

17q21 SNPs affect function of ORMDL3 intronic enhancer. We next wanted to predict the cis-regulatory elements (enhancers) that are affected by asthma-risk variants present in the $17 \mathrm{q} 21$ locus. Among the 23 SNPs that directly overlap 21 DHS in the $17 q 21$ locus (shown in Fig. 1e), we focused our analysis on two groups of SNPs: (i) those that overlap cis-regulatory elements (DHS) selectively enriched in immune cell types where ORMDL3 expression is affected (Fig. 3a, green highlighted boxes), and (ii) those that overlap DHS present in immune cell types as well as a wide range of other primary cell types (Fig. 3a, orange highlighted boxes). The latter SNPs could impact the function of multiple other cell types potentially involved in disease pathogenesis.

Three asthma-risk SNPs (rs4065275, rs8076131 and rs12603332) overlapped DHS in the first intron of ORMDL3
(Fig. 3a, top panel); this $\sim 3.0 \mathrm{~kb}$ intronic region was also highly enriched for the H3K27ac mark, suggestive of active enhancer activity, in $\mathrm{CD} 4{ }^{+} \mathrm{T}$ cells but not monocytes (Fig. 3a, bottom panel). Next, we asked if the activity of this ORDML3 intronic enhancer, as judged by enrichment levels of H3K27ac, was modified by the asthma-risk alleles. We utilized a large data set of $\mathrm{H} 3 \mathrm{~K} 27 \mathrm{ac}$ ChIP-Seq assays in memory CD4 ${ }^{+} \mathrm{T}$ cells to assess effects of asthma-risk genotype on enhancer activity. An increase ( $\sim 1.44$ fold) in H3K27ac enrichment was observed in subjects carrying the asthma-risk allele when compared with those carrying the non-risk allele (Fig. 3b), suggesting that the activity of this enhancer is altered by asthma-risk SNPs.

In addition to the ORMDL3 intronic enhancer, two other DHS regions that overlap asthma-risk SNPs were also present in $25-60 \%$ of the cell types analysed, that is were not strictly cell-type specific (Fig. 3a, top panel). These regions included the $Z P B P 2$ intronic region that overlaps rs12936231 and the intergenic region that is $25 \mathrm{~kb}$ downstream of ORMDL3 intergenic region overlapping ncSNP (rs4795408).

The three DHS sites that were selectively enriched in lymphocytes were present near the IKZF3 gene, some located well over $100 \mathrm{~kb}$ away from the ORMDL3 promoter. The distal IKZF3 intergenic region overlaps ncSNP (rs12946510) and similar to the ORMDL3 intronic enhancer, this region showed increased activity ( $\mathrm{H} 3 \mathrm{~K} 27 \mathrm{ac}$ enrichment) in subjects carrying the asthma-risk allele (Fig. 3b,c). The other two regions were $\left(5^{\prime}\right)$ proximal and intronic $I K Z F 3$ regions, which overlap the ncSNPs rs2313430 and rs4795397, respectively (Fig. 3b,c). Overall, our observations point to several potentially important enhancer elements in the $17 \mathrm{q} 21$ locus, especially the ORMDL3 intronic enhancer, whose function is affected by asthma-risk variants.

17q21 asthma-risk SNPs switch CTCF-binding sites. We performed an in silico analysis to identify the transcription factors whose binding to the cis-regulatory regions described above is perturbed by asthma-risk SNPs. Our evaluation pointed to perturbation of two CTCF-binding motifs as well as motifs of several other transcription factors such as IRF1, PAX4, TCF3 (Fig. 4a and Supplementary Data set 5). We focused on CTCF binding because it directly overlaps the ORMDL3 intronic enhancer region (Fig. 4a), and CTFC protein has been shown to play an important role in enhancer-promoter interactions and as an insulator protein $^{35}$. The first CTCF-binding motif was altered by an asthma-risk SNP (rs4065275) in the ORMDL3 intronic region causing an A-to-G change in the motif, which is predicted to enhance the binding of CTCF (Fig. 4a and Supplementary Data set 5). We experimentally confirmed that CTCF binding in the ORMDL3 intronic region was significantly enriched in both expanded and primary $\mathrm{T}$ cells from subjects carrying the risk allele (Fig. 4b). As a control region, we selected a well-known invariant CTCF binding site $\sim 50 \mathrm{~kb}$ proximal to ORMDL3 (near the GSDMA gene), which did not harbour any motif-disrupting asthma-risk SNP; this region did not show any significant change in CTCF binding (Supplementary Fig. 4a). In heterozygous subjects, we performed allele-specific analysis of CTCF-bound DNA and found strong preference for the risk allele (Fig. 4c and Supplementary Fig. 4b). Thus, we confirmed that the presence of asthma-risk SNP (rs4065275) introduces a CTCF-binding site in the ORMDL3 intronic region that harbours an active enhancer.

The second CTCF-binding motif was altered by asthma-risk SNP (rs12936231) in the ZPBP2 intronic region causing a G-to-C change in the CTCF motif, which was predicted in our in silico analysis and previously shown to impair CTCF binding ${ }^{27}$. We confirmed in $\mathrm{CD}^{+}{ }^{+}$and $\mathrm{CD} 8{ }^{+} \mathrm{T}$ cells that CTCF binding was almost completely lost in the $Z P B P 2$ intronic region from subjects 
a

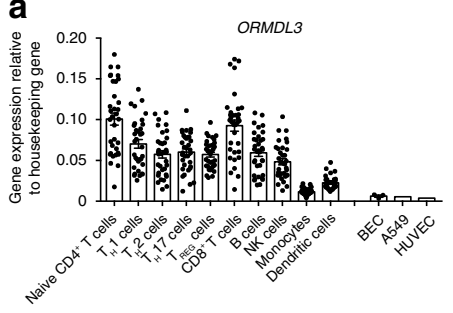

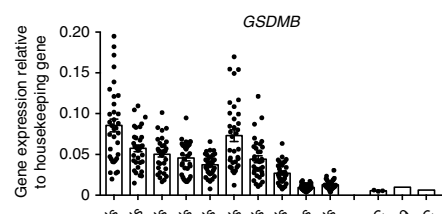

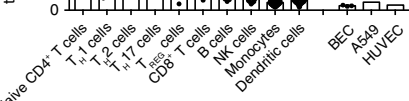

b
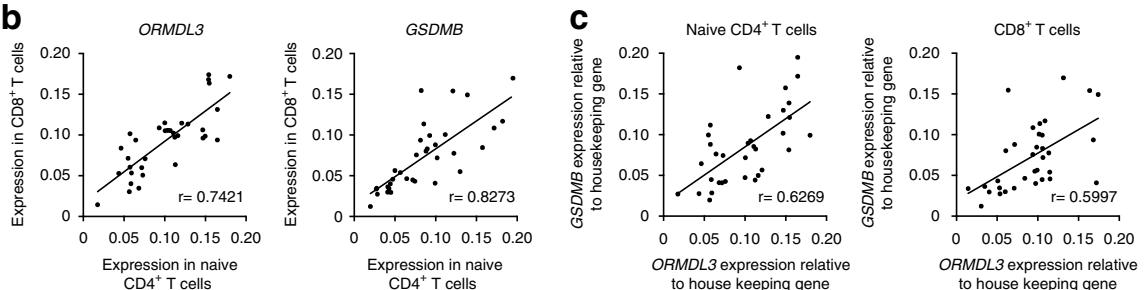

d
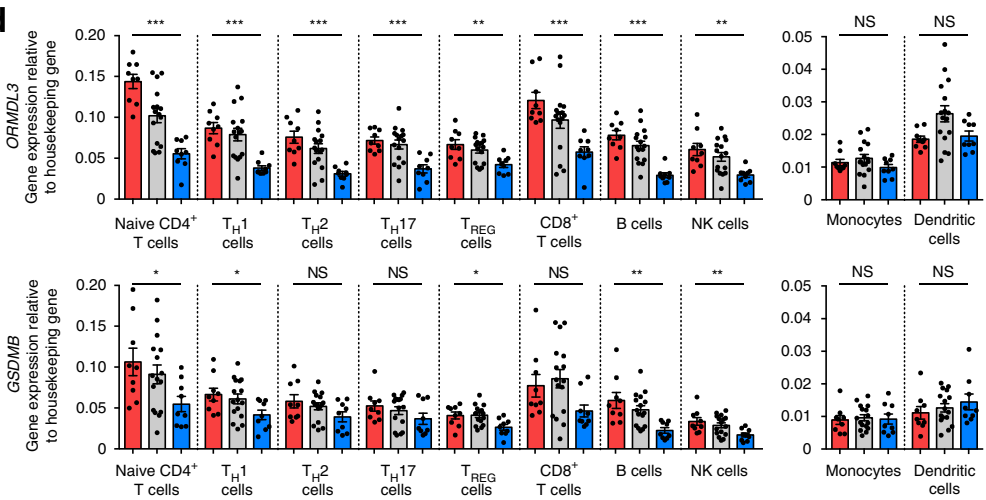

$\square$ Homozygous risk genotype (rs7216389 T/T) $\square$ Heterozygous genotype (rs7216389 T/C) $\square$ Homozygous non-risk genotype (rs7216389 C/C)
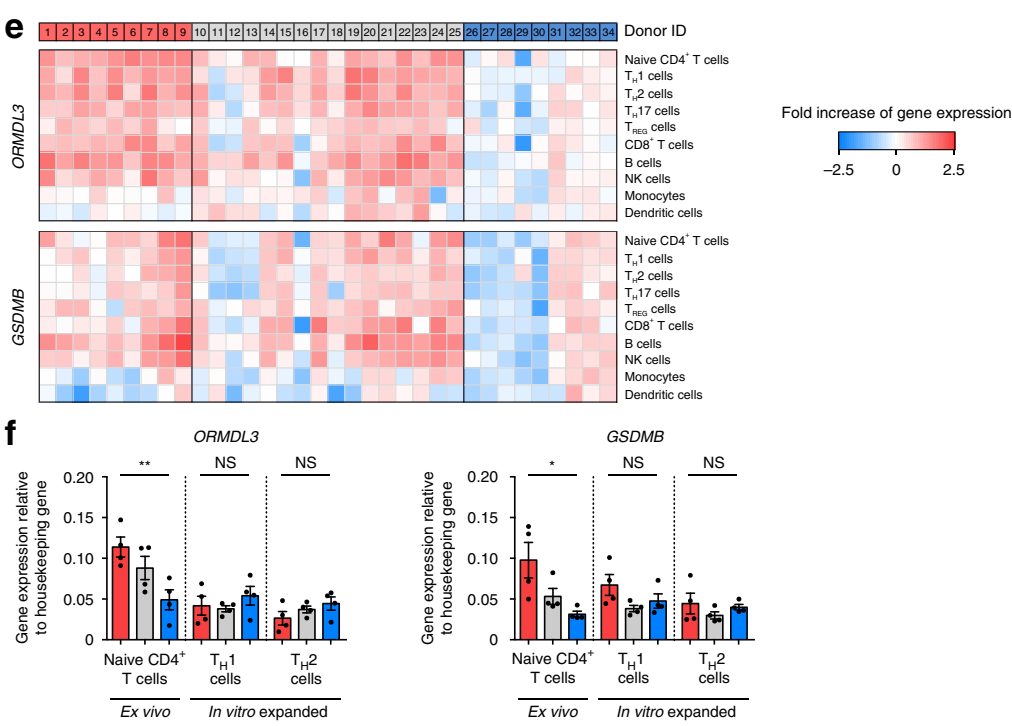

Figure 2 | 17q21 SNPs have pronounced effects on ORMDL3 expression in primary T and B cells. (a) Real-time PCR quantification of ORMDL3 and GSDMB transcript levels (relative to the housekeeping gene YWHAZ) in the indicated primary immune cell types $(n=34$ donors), bronchial epithelial cells (BEC; $n=3$ ), lung cancer cells ( $A 549 ; n=1)$ and human umbilical vein endothelial cells (HUVEC; $n=1)$. (b) Correlation of ORMDL3 and GSDMB transcript levels between naive CD4 ${ }^{+}$T cells and $\mathrm{CD} 8^{+}{ }^{+}$T cells. (c) Correlation between ORMDL3 and GSDMB transcript levels in naive CD4 ${ }^{+} \mathrm{T}_{\text {cells }}$ and $\mathrm{CD} 8{ }^{+}$ T cells. (d) ORMDL3 and GSDMB transcript levels in the indicated cell types from donors categorized based on the genotype for asthma-associated SNP rs7216389 (homozygous risk (T/T): $n=9$, red bar; heterozygous (T/C): $n=16$, grey bar; homozygous non-risk (C/C): $n=9$, blue bar). (e) Heat maps show Log2 fold change in transcript levels of ORMDL3 and GSDMB relative to the average expression levels seen in the corresponding cell types from donors with the non-risk (C/C) genotype; the donors are ordered based on genotype and the number indicates the unique ID (Supplementary Data set 3). (f) ORMDL3 and GSDMB transcript levels in naive CD4 ${ }^{+} \mathrm{T}$ cells and in in vitro expanded and polarized $\mathrm{T}_{\mathrm{H}}{ }^{1}$ or $\mathrm{T}_{\mathrm{H}} 2$ cells from matched donors (see Methods), categorized based on the genotype as in $\mathbf{d}$. Each dot represents data from a single donor. Error bars are mean \pm s.e.m.; ${ }^{\star} P<0.05$, ${ }^{\star \star} P<0.01$, and ${ }^{\star \star \star} P<0.001$ by Student's unpaired two-tailed $t$-test; NS, not significant; $r$ value indicates the Spearman correlation coefficient. 
a

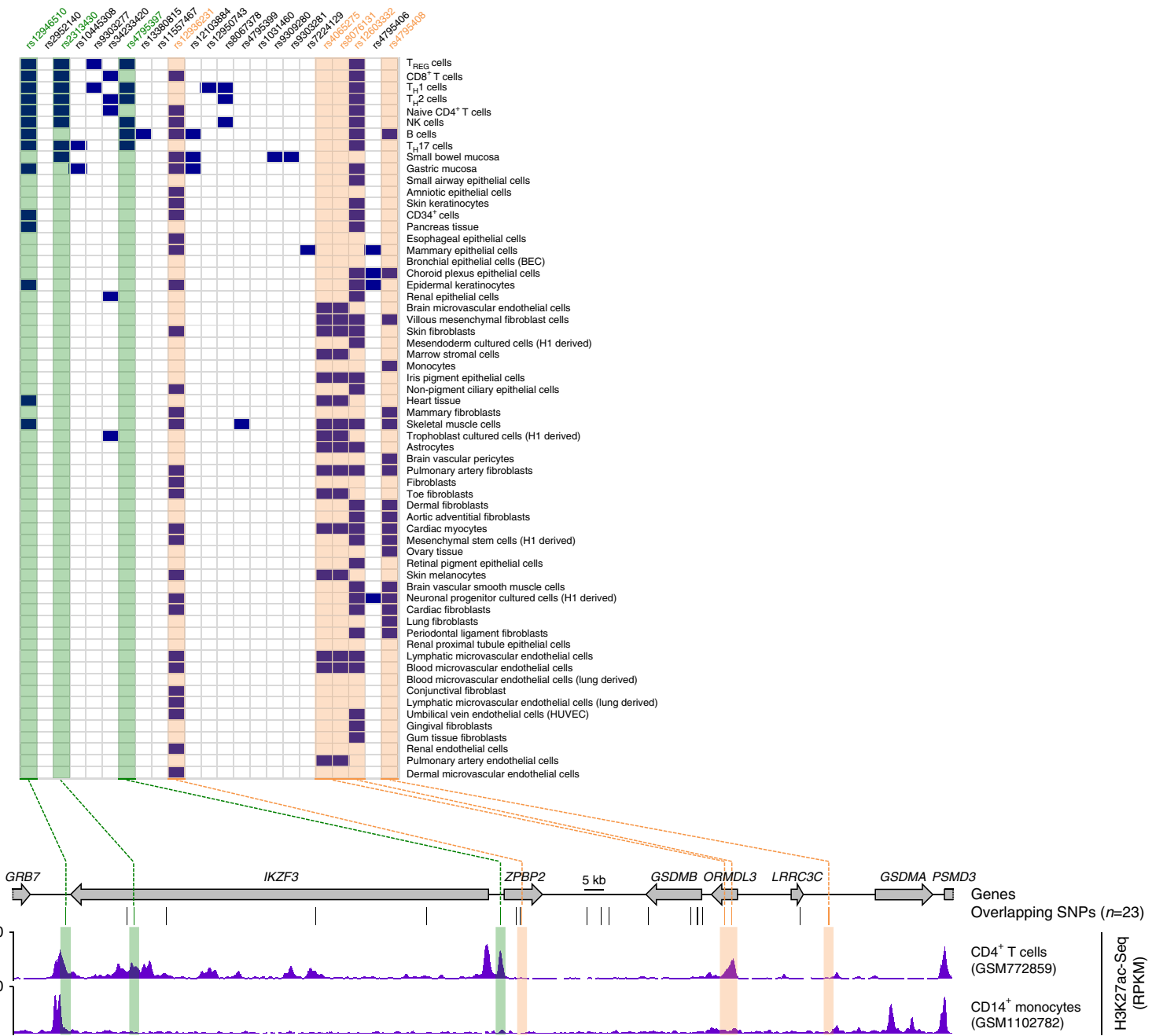

b
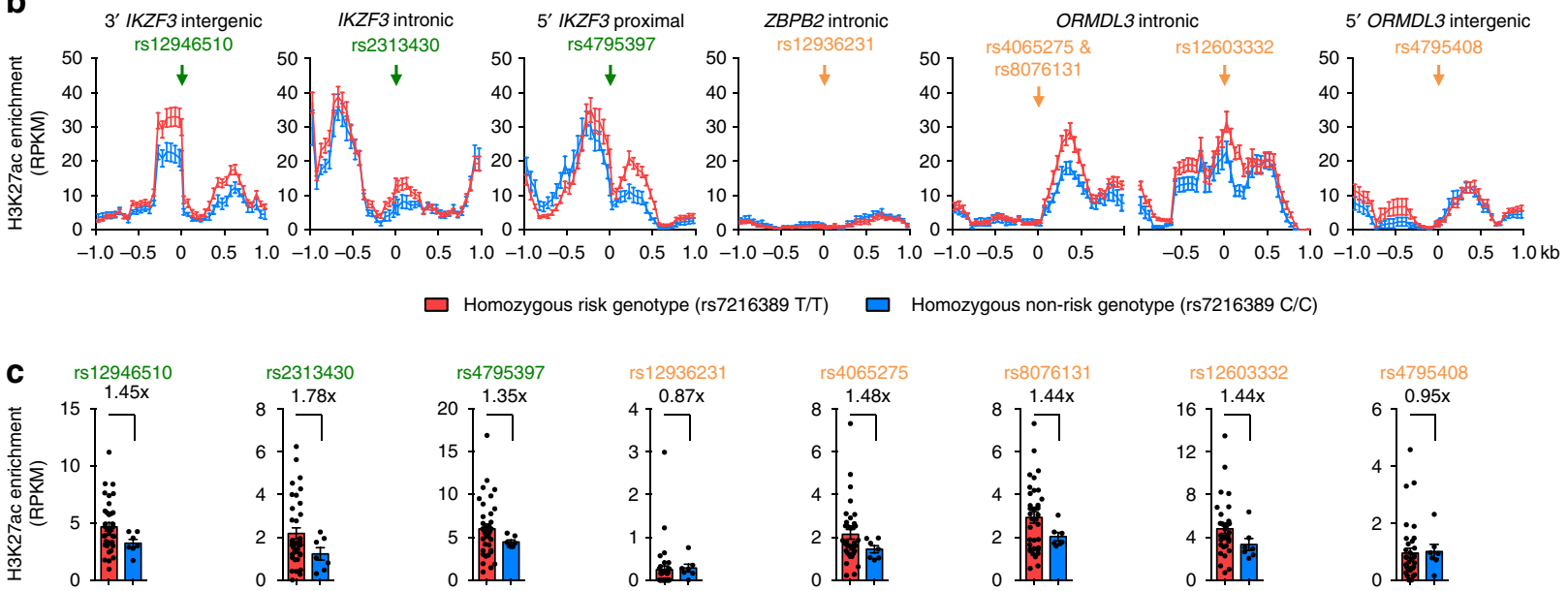

Figure 3 | 17q21 SNPs affect the function of an intronic enhancer in ORMDL3. (a) 17q21 SNPs (columns) that overlap with DHS (shown in dark blue squares) in different primary cell types (rows), as described in Fig. 1e,f. Green boxes highlight SNPs that overlap DHS enriched in lymphocytes and orange boxes highlight SNPs that overlap DHS in many immune and non-immune cell types. Bottom panel shows UCSC tracks of the 17q21 locus, along with asthma-associated SNPs that overlap DHS. H3K27ac enrichment tracks of CD4 ${ }^{+}$T cells and CD14 ${ }^{+}$monocytes (from NIH Epigenomics Roadmap) are shown below. Boxes highlight SNPs of interest that overlap DHS. (b) Tracks showing comparison of average H3K27ac enrichment values (RPKM) (in each 50-bp window spanning $1 \mathrm{~kb}$ region on either side of the SNPs of interest) between homozygous risk ( $r s 7216389 \mathrm{~T} / \mathrm{T}, n=36$ ) and non-risk (rs7216389 $\mathrm{C} / \mathrm{C}, n=7$ ) samples. Arrow indicates location of the SNP; error bars are mean \pm s.e.m. (c) H3K27ac enrichment values for a 200-bp region around the indicated asthma-risk SNP. Each dot represents data from a single assay; error bars indicate mean \pm s.e.m.; numbers indicate average fold change in H3K27ac enrichment between carries with risk and non-risk allele. 


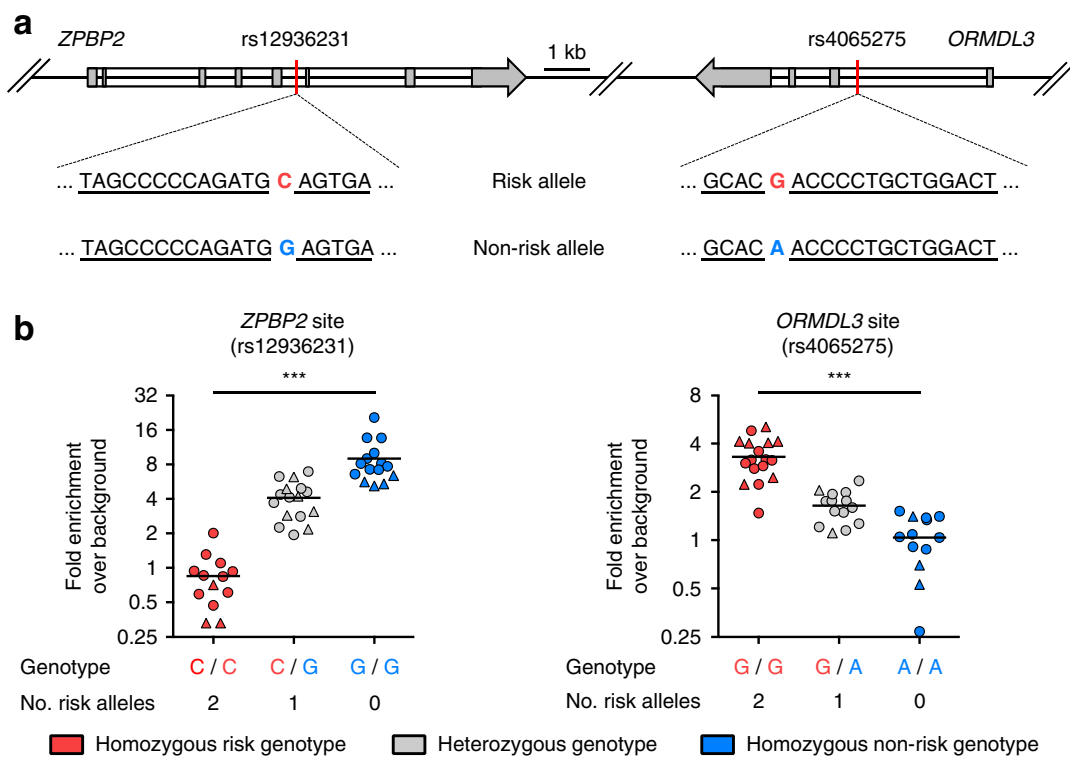

c
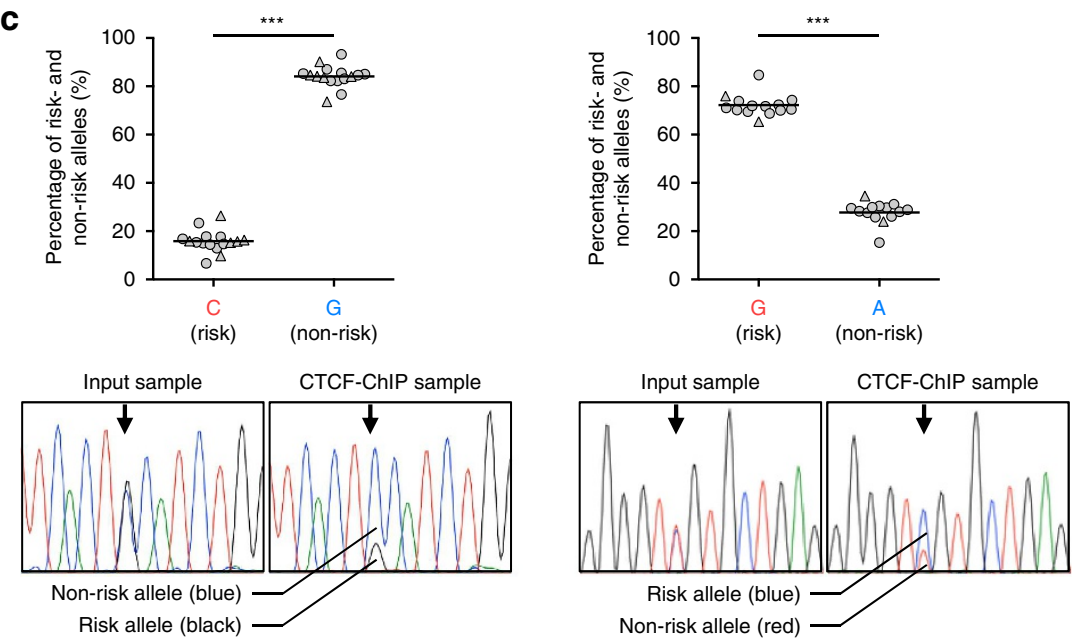

d ZPBP2 site (rs12936231)

GATG C AGTG.

Risk allele

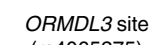

(rs4065275)

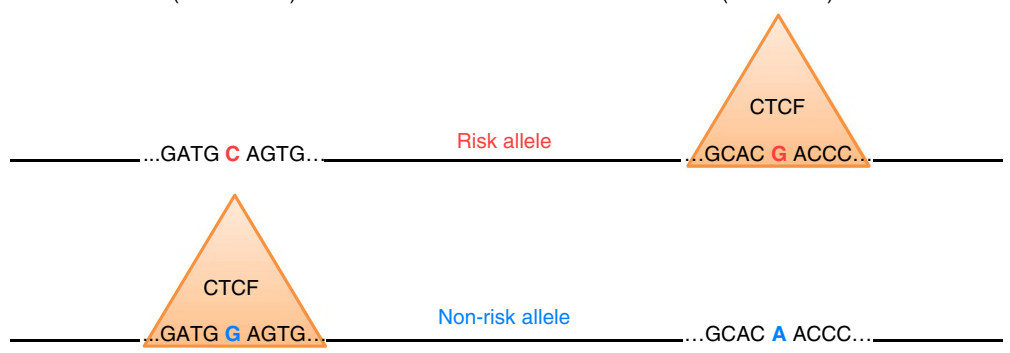

Figure 4 | CTCF-binding sites are switched by 17q21 SNPs. (a) Schematic representation of the 17q21 locus containing CTCF-binding motifs (underlined sequence) that overlap with the linked SNPs rs12936231 (C/G; ZPBP2 site) and rs4065275 (G/A; ORMDL3 site); the asthma-risk SNP is indicated in red, the non-risk SNP in blue. (b) Real-time PCR quantification of DNA sequences, containing the 17q21 linked SNPs rs12936231 (ZPBP2 site) or rs4065275 (ORMDL3 site), after anti-CTCF ChIP of chromatin extracts obtained from polarized $\mathrm{T}_{\mathrm{H}} 1, \mathrm{~T}_{\mathrm{H}} 2$ cells (shown as circles) and primary CD8 ${ }^{+} \mathrm{T}$ cells (shown as triangles; see Methods) of donors categorized based on allelic status of SNP overlapping the respective CTCF motif (as shown in a). Data are expressed as fold enrichment relative to an irrelevant background control; data were obtained from four independent experiments and each dot represents data from a single ChIP assay; $n=44$ assays from 15 subjects (see Methods and Supplementary Fig. 4c,d). (c) Percentage of DNA sequences containing the risk and non-risk SNPs at rs12936231 (ZPBP2 site) or rs4065275 (ORMDL3 site) following Sanger sequencing of DNA obtained following anti-CTCF ChIP assay performed in heterozygous donors shown in $\mathbf{b}$ (see Methods and Supplementary Fig. 4b); shown below are nucleotide traces from Sanger sequencing of ChIP and input DNA from a representative experiment. (d) Schematic representation of the switch in CTCF binding introduced by the linked asthma-risk SNPs (rs12936231 and rs4065275) in the 17q21 locus; orange triangles represent binding of CTCF to the preferred allele. ${ }^{\star \star \star} P<0.001$ by Mann-Whitney U-test. 
carrying the asthma-risk SNP, and this finding was true at the allele-specific level (Fig. 4b,c). Thus, the asthma-risk haploblock harbours linked SNPs that switch the binding site of CTCF from the ZPBP2 to the ORMDL3 intronic region (Fig. $4 \mathrm{~d}$ ).

17q21 SNPs modify ORMDL3 promoter-enhancer interactions. Since CTCF has a major role in long-range interactions of cis-regulatory elements to cognate promoter regions ${ }^{36-39}$, we hypothesized that the switching of CTCF-binding sites may affect the $3 \mathrm{D}$ architecture (organization) of the $17 \mathrm{q} 21$ locus to favour enhanced transcription of ORMDL3 in the asthma-risk alleles. In order to define all the distal cis-regulatory elements that potentially regulate $O R M D L 3$ expression in an asthma-risk genotype-dependent manner, we performed 4C-Seq assays (see Methods) in primary $\mathrm{CD}^{+} \mathrm{T}$ cells from subjects homozygous for the risk $(n=4)$ and non-risk alleles $(n=4)$. By designating the ORMDL3 promoter as the bait for $4 \mathrm{C}$, we detect at a high resolution the physical interactions of any potential cisregulatory element in the extended $17 \mathrm{q} 21$ locus with the ORMDL3 promoter region (Fig. 5a,b). The $4 \mathrm{C}$-Seq assay ${ }^{40,41}$ conditions were initially optimized in a human $\mathrm{T}$ cell line (HUT-78) to robustly detect long-range interactions of the ORMDL3 promoter (Supplementary Fig. 5a and see Methods).

Genomic regions interacting with the ORMDL3 promoter region were determined using two complementary analysis methods for $4 \mathrm{C}$ data (4C-ker ${ }^{42}$ and 4 Cseqpipe ${ }^{40}$, see Methods). We first pooled data from all risk and non-risk subjects and identified several interacting regions including the CTCF-bound regions near $Z B P B 2$ and $O R M D L 3$ gene loci, the $I K Z F 3$ promoter region and a number of other potential enhancer regions in the IKZF3 intronic region (Fig. 5c), suggesting extensive long-range interactions of the ORMDL3 promoter region in primary human $\mathrm{CD}^{+}{ }^{+} \mathrm{T}$ cells. Most strikingly, the IKZF3 promoter region and other potential enhancers in the $I K Z F 3$ intronic region that were enriched for H3K27ac and H3K4me1 marks in CD4 ${ }^{+} \mathrm{T}$ cells but not monocytes (that is, selectively active in $\mathrm{CD} 4{ }^{+} \mathrm{T}$ cells) appeared to interact with the ORMDL3 promoter region exclusively in subjects carrying the $17 \mathrm{q} 21$ asthma-risk allele (Fig. 5c, red highlighted boxes; for example, region chr17: $38,022,212-38,022,898$ (hg19) with an adjusted $P$ value of 0.000736 using the $4 \mathrm{C}$-ker method ${ }^{42}$, Supplementary Data set 6 ), whereas the ORMDL3 promoter region of non-risk alleles interacted with the CTCF-binding site in the ZPBP2 intronic region (Fig. 5c, blue highlighted boxes), which presumably insulated (blocked) the interactions to the adjacent active IKZF3 cis-regulatory elements. Though subjects in each group showed some variability, potentially due to their genotypic variation within this locus as we classified subjects as risk and non-risk only based on genotype at rs7216389, rs4065275 and rs12936231 (see Methods), the overall pattern was consistent across most donors (Supplementary Fig. 5b and Supplementary Data sets 6 and 7).

Our results suggest that the $3 \mathrm{D}$ organization of the $17 \mathrm{q} 21$ locus is modified in memory $\mathrm{CD}^{+} \mathrm{T}$ cells from subjects with the 17 q21 asthma-risk alleles to favour selective recruitment of the IKZF3 cis-regulatory elements to the ORMDL3 promoter region (Fig. 5d), which correlated with its increased transcriptional activity.

ORMDL3 negatively regulates IL-2 production by $\mathrm{CD} 4{ }^{+} \mathrm{T}$ cells. Next, we wanted to determine the functional consequences of altered levels of ORMDL3 transcripts in primary CD4 ${ }^{+} \mathrm{T}$ cells, as $17 q 21$ SNPs had the greatest impact on ORMDL3 expression in this cell type. ORMDL3 is an endoplasmic reticulum membrane protein, and studies in cell lines and in epithelial cells have indicated its involvement in $\mathrm{Ca}^{2+}$ signalling, sphingolipid metabolism and unfolded-protein responses ${ }^{43-45}$; however, its function in primary $\mathrm{T}$ cells has not been fully investigated. Here we sought to determine the functional role of ORMDL3 in memory CD4 ${ }^{+}$ $\mathrm{T}$ cells with a specific focus on cytokines released following activation. Freshly isolated human memory $\mathrm{CD}^{+} \mathrm{T}$ cells were transfected with small interfering RNA (siRNA) pools targeting the ORMDL3 transcript, cultured for $48 \mathrm{~h}$ to achieve adequate gene knockdown, and then activated by $\mathrm{T}$ cell receptor ligation (anti-CD3) and co-stimulation (anti-CD28) to study effects on cytokine production (Fig. $6 \mathrm{a}$ and see Methods).

We achieved over $50 \%$ reduction in ORMDL3 transcript levels without affecting cell viability due to transfection (Fig. $6 \mathrm{~b}$ and Supplementary Fig. 6a,c). Among the cytokines and chemokines measured, the strongest effect ( $>40 \%$ change) was seen for IL-2 production, which was significantly increased in the ORMDL3 siRNA-treated conditions compared to control siRNA conditions (Fig. 6c). IL-2 production was not affected by knocking down GSDMB or other control genes such as TBX21 and GATA3 (Fig. 6d).

At the transcriptional level, we observed similar effects on activation-induced expression of IL2 transcripts following ORMDL3 knockdown (Fig. 6e). Notably, we observed significant effects at very early $(3,6 \mathrm{~h})$ and late $(18 \mathrm{~h})$ time points following stimulation, suggesting sustained effects of ORMDL3 knockdown (Fig. 6e, right panel). We also confirmed these effects at the protein level using fluorescence-activated cell sorting (FACS)based intracellular cytokine detection assay (Fig. 6f). We next asked if the negative regulatory effect on IL2 transcription was also observed in unmanipulated $\mathrm{T}$ cells from subjects with varying levels of ORMDL3 expression. As expected, we found a striking correlation between baseline ORMDL3 expression and the levels of IL2 transcripts produced following brief ex vivo stimulation of memory $\mathrm{CD}^{+} \mathrm{T}$ cells with phorbol myristate acetate (PMA) and Ionomycin (Fig. 6g), suggesting that this correlation may also hold in vivo. Altogether, our results provide strong evidence that ORMDL3 negatively regulates IL-2 production in $\mathrm{CD}^{+} \mathrm{T}$ cells, and that overexpression of ORMDL3 in subjects carrying the asthma-risk alleles is likely to reduce IL-2 production in vivo, which in turn may have important functional effects that contribute to the development of asthma ${ }^{46}$

ORMDL3 knockdown also led to modest changes in the production of other cytokines such as interferon- $\gamma$ (IFN- $\gamma$ ), IL-10 and IL-13, suggesting that ORMDL3 is likely to have a broader spectrum of functions in T cells. Knocking down GSDMB led to modest changes in the production of TNF, IL-13 and IL-16 (Fig. 6h), suggesting that the product of this gene is also likely to affect the function of $T$ cells. Overall, our results suggest that physiological alteration in the levels of ORMDL3 and GSMDB induced by the asthma-risk allele may be sufficient to modulate the functional capacity of $\mathrm{T}$ cells.

\section{Discussion}

The overwhelming majority of the sequence variants (SNPs) associated with disease-risk haplotypes in GWAS studies do not change protein coding, implying that a proportion of them may be located in regulatory regions which act in cis to alter gene expression $1,16,20,47$. Since in many instances cis-regulatory regions are highly cell type-specific, the effects of SNPs that affect their function are also likely to be more pronounced in just a few cell types. The failure to link non-coding risk SNPs to the cell types in which they affect function has significantly hampered the progress of functional studies aimed at identifying biological effects of GWAS SNPs. In this study, we attempted to solve this problem by taking a comprehensive approach to predict and test the effects of $17 \mathrm{q} 21$ asthma-risk SNPs on expression of ORMDL3 in various cell types. We show that 


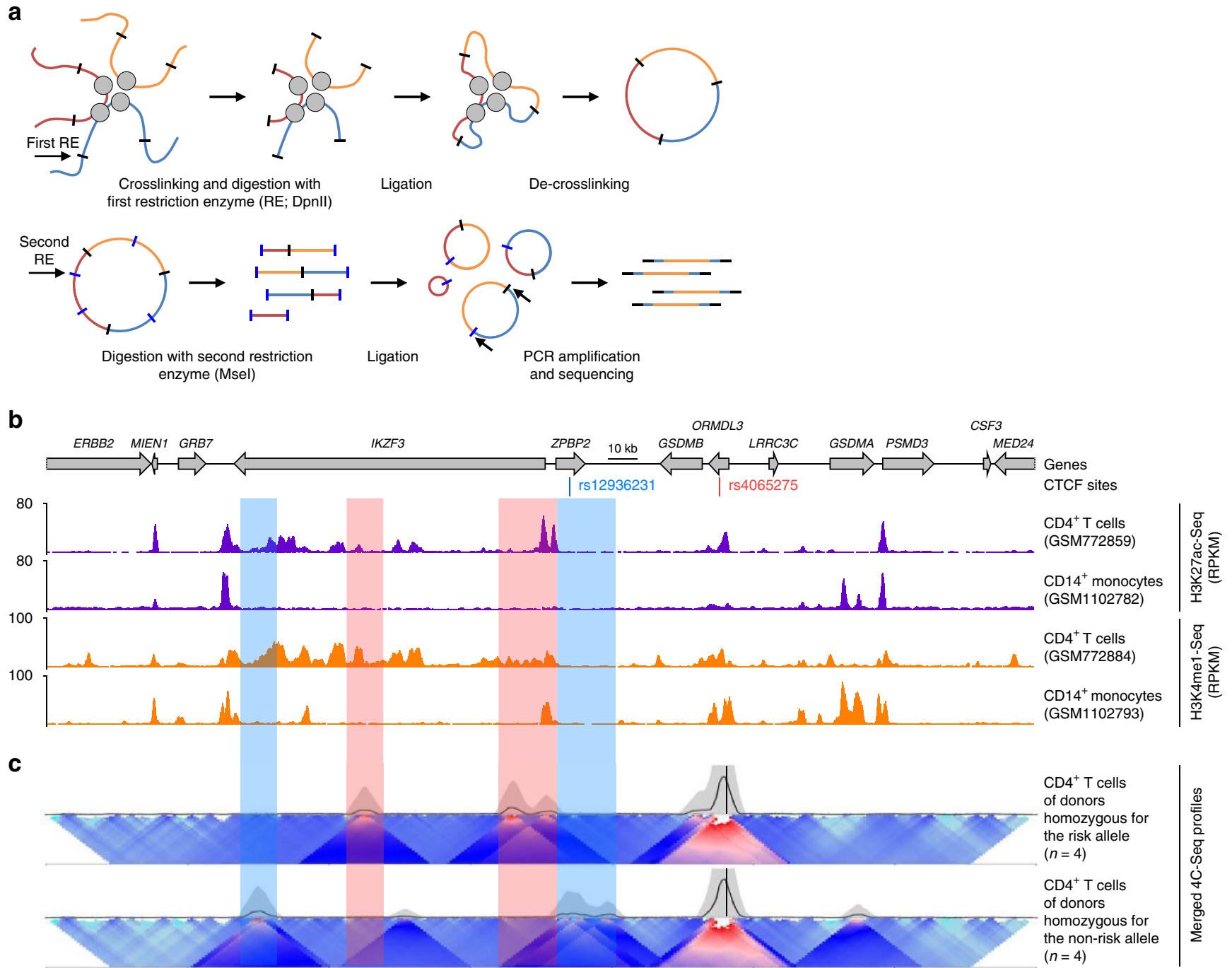

d

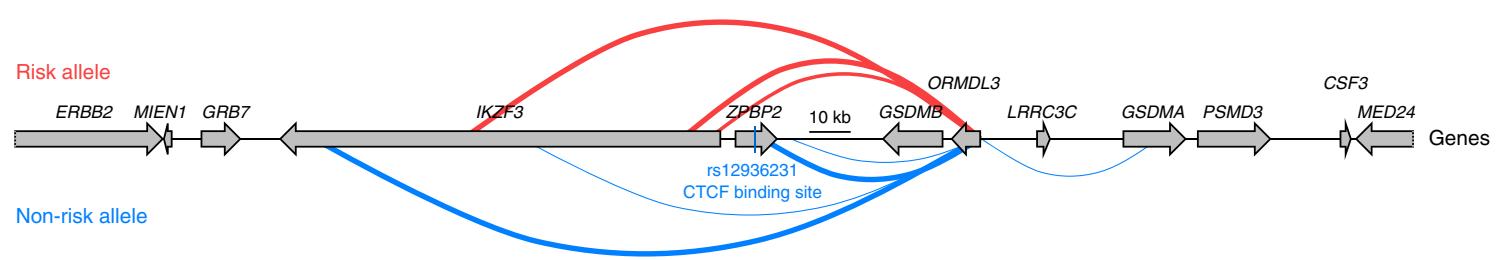

Figure 5 | Asthma-risk SNPs modify long-range ORMDL3 promoter-enhancer interactions. (a) Schematic representation of the 4C-Seq assay and the choice of restriction enzymes used for various steps. (b) UCSC gene tracks of 17q21 locus (chr17: 37,849,238 - 38,189,238 (hg19); 340 kb) showing the CTCF-binding sites that overlap with the linked SNPs rs12936231 (C/G; ZPBP2 site) and rs4065275 (G/A; ORMDL3 site), along with H3K27ac and $\mathrm{H} 3 \mathrm{~K} 4 \mathrm{me} 1$ enrichment tracks of CD4 ${ }^{+} \mathrm{T}$ cells and CD14 ${ }^{+}$monocytes. (c) Merged $4 \mathrm{C}$-Seq domainograms, using colour-coded intensity values to indicate relative levels of interactions (red denotes the strongest interactions and dark blue to turquoise representing gradually decreasing frequencies), generated using 4Cseqpipe ${ }^{40}$ (see Methods) are displayed for CD4 ${ }^{+}$T cells from subjects homozygous for the risk $(n=4)$ and non-risk alleles $(n=4)$; the bait region (ORMDL3 promoter) is marked as a black line. Shaded boxes highlight regions that interact with the bait region at the ORMDL3 promoter in the risk and non-risk alleles (shown in red and blue colour, respectively). (d) Schematic representation of the DNA regions interacting with the ORMDL3 promoter in the asthma-risk (red colour) and non-risk alleles (blue colour), the CTCF-binding site in the ZPBP2 region of the non-risk alleles in shown as a blue line.

primary immune cell types, with the exception of monocytes and dendritic cells, but not lung structural cells such as BECs, are most sensitive to the effects of $17 \mathrm{q} 21$ SNPs. Notably, this approach allowed us to narrow down the cell types in which ORMDL3 potentially influences disease outcomes, and, therefore, perform detailed functional studies in a relevant cell type (primary human CD ${ }^{+} \mathrm{T}$ cells) and show that ORMDL3 negatively regulates the transcription of $I L 2$. Given the pleotropic role of IL-2 in modulating the differentiation and function of $\mathrm{T}_{\mathrm{H}}$ cell subsets, it is likely that this effect on IL-2 production could be pivotal for driving genetic risk for asthma and autoimmunity.

Previous studies have examined the function of the conserved ORM family proteins in yeast ${ }^{48,49}$ (which includes ORMDL3 in humans), as well as ORMDL3 itself in various mammalian cell lines and in primary cell types relevant to asthma pathogenesis 
such as epithelial cells, eosinophils, mast cells and whole PBMCs ${ }^{23,43-45,50-54}$. ORM family proteins were originally shown to mediate sphingolipid metabolism in yeast ${ }^{48,49}$, which was subsequently confirmed in mammalian cells ${ }^{44}$. As an endoplasmic reticulum membrane protein, ORMDL3 has been shown to be involved in endoplasmic reticulum-mediated $\mathrm{Ca}^{2+}$ homeostasis by depleting $\mathrm{Ca} 2{ }^{+}$stores of the endoplasmic reticulum through an interaction with the SERCA pump in the endoplasmic reticulum ${ }^{43,50}$. In this context, ORMDL3 was shown to diminish the translocation of the nuclear factor of activated a

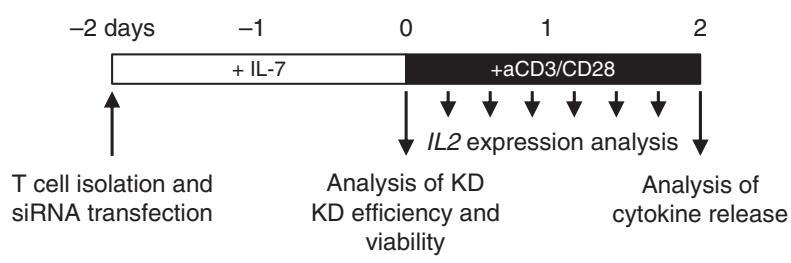

b

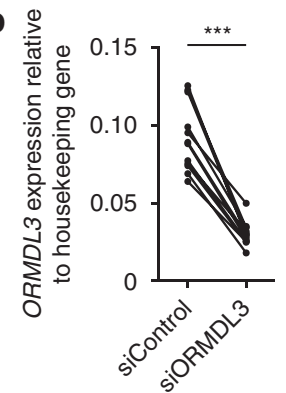

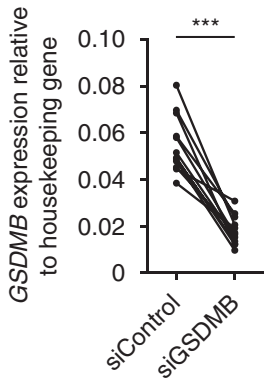

d

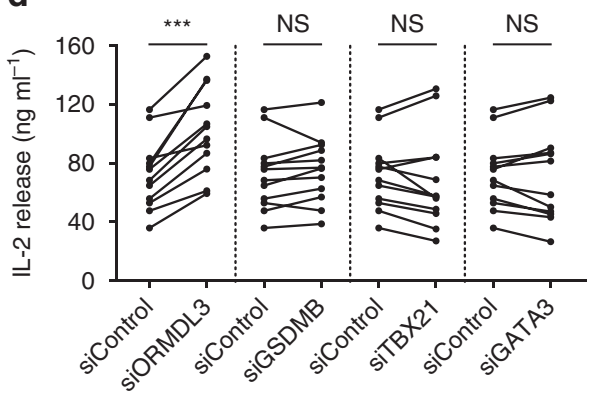

e

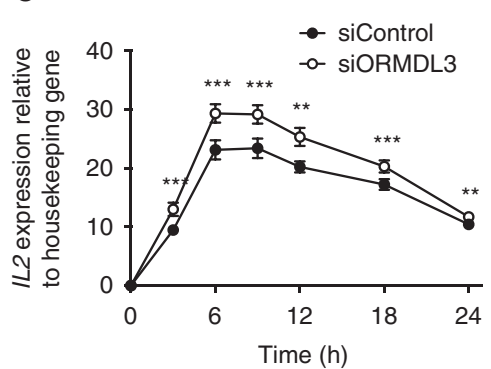

f

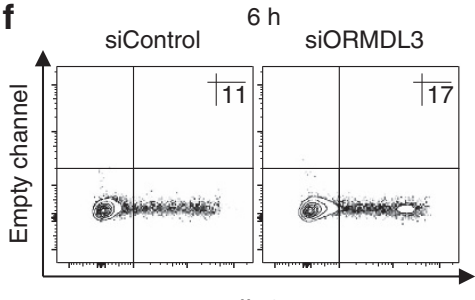

g

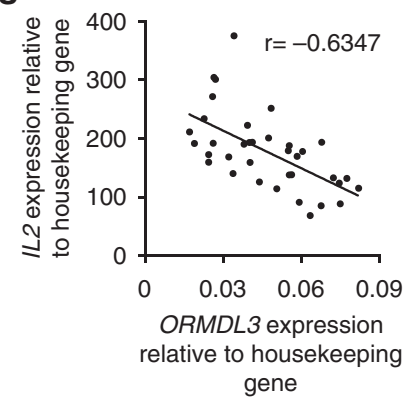

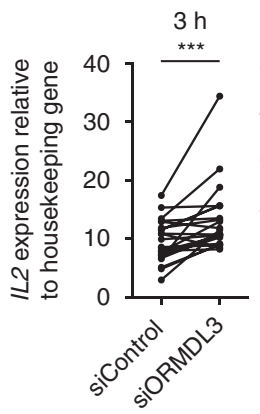
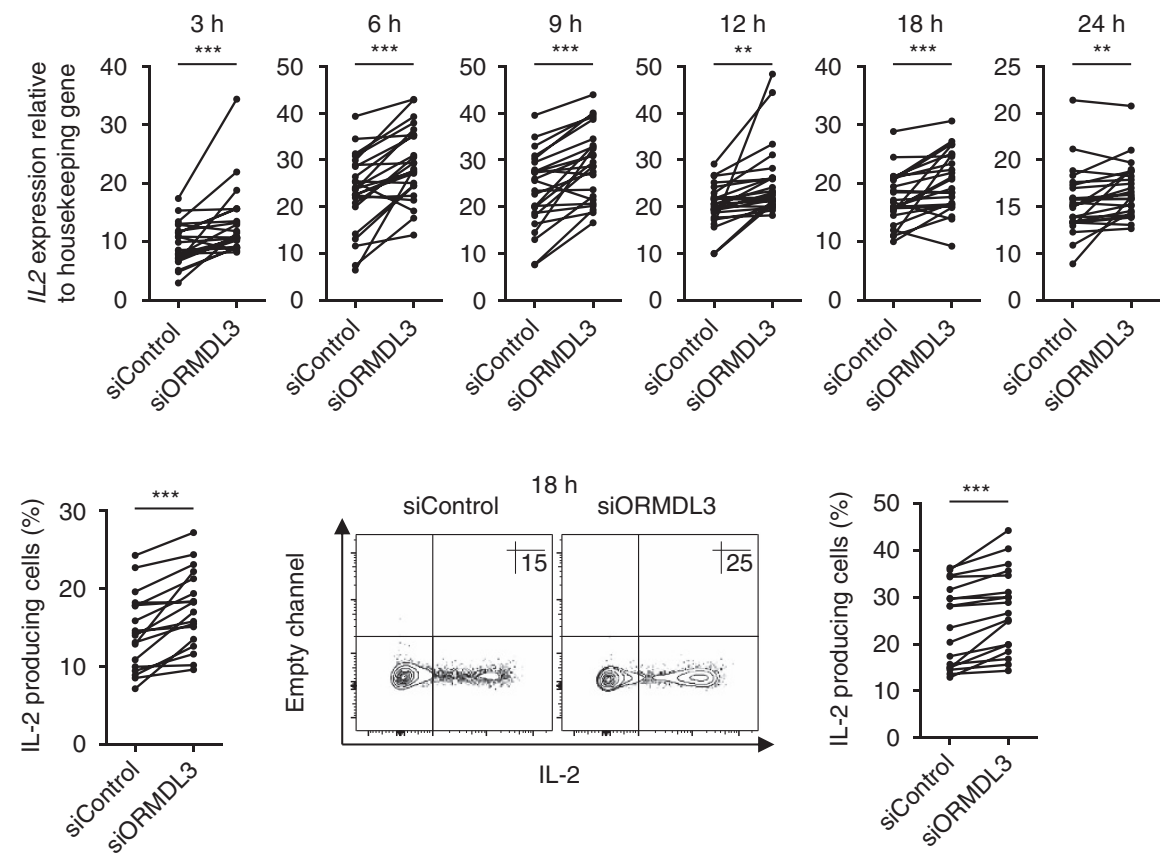

h

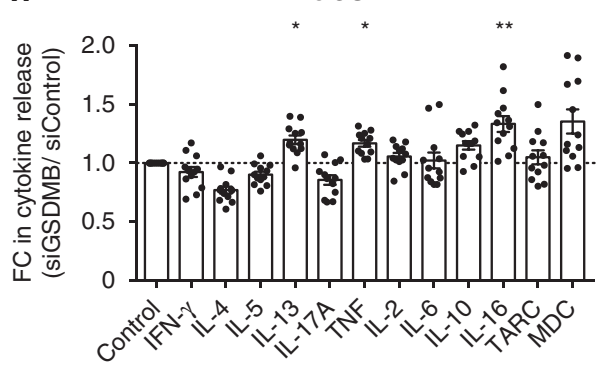


$\mathrm{T}$ cells (NFAT) in Jurkat cell lines and modestly reduce cytokine release after stimulation ${ }^{50}$. Therefore, it is likely that the negative regulatory effects of ORMDL3 on IL-2 production in primary T cells are also due to perturbation of $\mathrm{Ca}^{2+}$ signalling and NFAT function following $\mathrm{T}$ cell activation.

Overexpression of ORMDL3 in BECs has been shown to activate components of endoplasmic reticulum stress or unfolded protein response, resulting in increased expression of several pro-inflammatory molecules involved in airway remodelling and inflammation ${ }^{45,51}$. Recent studies in murine primary eosinophils and mast cells have revealed opposing functions in these cell types; in eosinophils ORMDL3 positively regulates its activation and function by influencing cell shape change, adhesion and recruitment to sites of inflammation in vivo, while in mast cells ORMDL3 negatively regulates activation-induced expression of pro-inflammatory mediators and chemotactic responses ${ }^{51,53}$. Thus, it appears that the function of ORMDL3 may vary considerably depending on the cell type, and, therefore, it will be important to first know whether the 17q21 asthma-risk SNPs in fact affect the expression of ORMDL3 in these cell types; this information will allow determination of whether these cell types are the dominant factors driving the 17q21-related genetic risk of asthma.

While all SNPs in genetic linkage may be statistically significantly associated with asthma, only a subset will be functionally relevant. For instance, the $17 \mathrm{q} 21$ locus has a dense haploblock of 136 SNPs linked to asthma risk, a number too large to allow exploration of each SNP's potential function. In determining which SNPs are likely to influence pathophysiology, our basic assumption is that a significant fraction of ncSNPs that are deemed to be functional in asthma act on cis-regulatory elements that are important for controlling the expression of $17 \mathrm{q} 21$ genes. Therefore, to identify functional SNPs in the 17q21 haploblock, we first focused on SNPs that overlap cis-regulatory regions that are active in several cell types, especially those (for example, $\mathrm{CD} 4{ }^{+} \mathrm{T}$ cells) in which expression of ORMDL3 is affected by the 17q21 SNPs, and then tested whether the presence of these SNPs alters epigenetic profiles, that is, activity at relevant cis-regulatory DNA regions. We identified an enhancer region in the first intron of the ORMDL3 gene that overlapped three 17q21 asthma-risk SNPs (rs4065275, rs8076131, and rs12603332), and this region showed increased activity in subjects carrying the asthma-risk allele. The rs4065275 variant introduced a binding site for the multi-functional zinc finger protein CTCF, whereas the linked rs12936231 variant, about $55 \mathrm{~kb}$ downstream of ORMDL3, disrupted a second CTCF-binding site, which together results in interchanging of two CTCF sites in the vicinity of the ORMDL3 gene locus.

CTCF binds insulator regions in the genome ${ }^{55-57}$, where it forms gene boundaries by blocking interactions between distal enhancers and promoters; when present in introns, it influences the efficiency of pre-mRNA splicing at weak splice sites by altering the processivity of RNA polymerase II (pol II ${ }^{35}$; however, we did not observe any changes in splicing of ORMDL3 transcripts (data not shown). More recently, genomewide analysis of CTCF-binding patterns coupled with long-range chromatin interaction studies (ChIA-PET) have shown that CTCF has a pivotal role in promoting interaction of multiple enhancers to cognate gene promoters ${ }^{58}$. Therefore, we hypothesized that the switching of CTCF sites by asthma-risk SNPs may alter the three-dimensional structure of chromatin in the 17q21 locus and promote the interaction of active enhancer regions with their cognate promoter (ORMDL3), thereby increasing ORMDL3 expression in carriers of the asthma-risk SNPs. Experimentally, we confirmed genotype-dependent differential looping (altered 3D organization) in the 17q21 locus that resulted in the recruitment of active cell-specific cis-regulatory elements (for example, of the $5^{\prime}$ IKZF3 proximal region which overlaps the asthma-risk SNP rs4795397) to the ORMDL3 promoter in asthma-risk alleles. Depending on the composition of the haploblock for a given donor (combination of SNPs), the differential looping may also recruit enhancer regions that themselves are affected by SNPs and thus creating variable and donor-specific reorganization of the $17 \mathrm{q} 21$ locus. These mechanisms may contribute to the exquisite cell type-specific effects of 17q21 SNPs on gene expression in people with various genotypes.

The discovery of new drug targets in a human disease requires identification of the pathogenic cell type(s) as well as the molecular factors and pathways that contribute to the disease. Our current knowledge of these points is incomplete for many diseases. The approach we have taken to improve our understanding of the genetic basis of asthma and autoimmunity aims to address some of these issues and may be applied to various other disease-associated genetic loci to unravel disease-relevant cell types and molecular pathways.

\begin{abstract}
Methods
Study subjects and sample processing. Blood samples from 34 donors were obtained from LJI's Normal Blood Donor Program after written informed consent Ethical approval for the use of this material was obtained from the Institutional Review Board (IRB) of the LJI. Only for the data shown in Fig. 3b,c, additional samples were obtained from another cohort of 38 donors (only subjects homozygous for either the asthma risk- or non-risk variant at rs7216389), after written informed consent. The genotyping of the 17q21 SNPs rs7216389, rs12936231 and rs4065275 was performed using the automated Sanger DNA sequencing platform (GENEWIZ, Inc.) with the primers listed in Supplementary Table 1. Details of the genotype of 17q21 SNPs, age and gender of study subjects are provided in Supplementary Data set 3.

For isolating immune subtypes from peripheral blood samples, PBMCs were first separated into a CD4 ${ }^{+}$memory cell fraction and remaining cells by use of the Memory CD4 ${ }^{+} \mathrm{T}$ Cell Isolation Kit (Miltenyi Biotec). Both populations were stained with cocktails of fluorescently conjugated antibodies (see Supplementary Table 2) and sorted on a FACSAria-II (Becton Dickinson) using the gating strategy shown in Supplementary Fig. 2. Sorted cells were washed, and directly lysed in
\end{abstract}

Figure 6 | ORMDL3 negatively regulates IL-2 production by CD4 ${ }^{+}$T cells. (a) Experimental design used for assessing effects of knocking down genes of interest in memory CD4 ${ }^{+}$T cells. (b) Real-time PCR quantification of ORMDL3 and GSDMB transcript levels (relative to the housekeeping gene YWHAZ) in memory $\mathrm{CD} 4{ }^{+}$T cells $48 \mathrm{~h}$ after knock down with control siRNA pools, ORMDL3 or GSDMB siRNA pools $(n=12$ donors). (c) Effects of ORMDL3 knockdown on cytokine release by memory $\mathrm{CD} 4^{+}$T cells activated for $48 \mathrm{~h}$ with antibodies to CD3 and CD28; data are expressed as fold change (FC) relative to control siRNA-treated conditions; error bars indicate mean \pm s.e.m. (d) Absolute values of IL-2 protein levels in culture supernatants from cells treated with the indicated siRNA pools. Data are presented as means of biological duplicates. (e) Time course of IL2 mRNA expression in activated memory CD4 ${ }^{+}$T cells $(n=24)$ following treatment with ORMDL3 (open circles) or control siRNA pools (closed circle); data from each donor for different time points after stimulation is shown in the right panel. (f) Representative FACS plots showing intracellular staining of IL-2 in memory CD4 ${ }^{+} \mathrm{T}$ cells activated for 6 or $18 \mathrm{~h}$ (after knockdown with siRNA pool for ORMDL3 or control siRNA); percentage of IL-2 producing cells in each donor is shown to the right $(n=18)$. ( $(\mathbf{g})$ Correlation of the levels of ORMDL3 transcripts (measured at baseline) and IL2 transcripts produced following ex vivo stimulation of memory CD4 ${ }^{+}$T cells $(n=36)$ with phorbol myristate acetate (PMA) and lonomycin for $4 \mathrm{~h}$. (h) Effects of GSDMB knockdown on cytokine release by memory CD4 ${ }^{+}$T cells, as described in c. Each dot represents data from a single donor. $r$, Spearman correlation coefficient; ${ }^{\star} P<0.05$, ${ }^{\star \star} P<0.01$, and ${ }^{\star \star \star} P<0.001$ by Student's paired two-tailed $t$-test, and following Bonferroni correction for multiple testing in $\mathbf{c}, \mathbf{d}$ and $\mathbf{h}$ (see Methods). 
TRIzol solution (Invitrogen) for subsequent isolation of total RNA or fixed for ChIP assays as described previously ${ }^{14}$.

Gene expression studies. Total RNA was extracted using the miRNeasy Micro Kit (Qiagen); complementary DNA was reverse-transcribed with the SuperScrip III First-Strand Synthesis System (Life Technologies). Real-time PCR employed the Fast Start Universal SYBR Green Master Mix (Roche); see Supplementary Table 1 for primer sequences. Data were acquired on the StepOnePlus Real-Time PCR System (Applied Biosystems); all results are presented in arbitrary units relative to expression of the housekeeping gene YWHAZ ${ }^{59}$

\section{Micro-scaled ChIP-Seq for H3K27ac and bioinformatics analysis. Samples} from an independent cohort of 38 donors (as described above) were utilized for this analysis (Supplementary Data set 3). A total of 43 ChIP-Seq assays (including 5 technical replicates) were performed as described previously ${ }^{14,60}$. Briefly, purified chromatin from $1 \times 10^{5} \mathrm{~T}_{\mathrm{H}} 2$ cell enriched $\mathrm{CD} 4{ }^{+}$memory subset $\left(\mathrm{CD} 3^{+} \mathrm{CD}^{+}\right.$ $\mathrm{CD}^{-} 5^{-} \mathrm{CD} 45 \mathrm{RA}^{-} \mathrm{CCR}^{+}$) was immunoprecipitated with a polyclonal antiH3K27ac antibody (Lot \#GR184333-1; ab4729; Abcam). Chromatin was incubated with $0.5 \mu \mathrm{g}$ antibody pre-coated to $5 \mu \mathrm{l}$ of protein A-coated magnetic beads (Invitrogen). Immunocomplexes were captured, washed and eluted; DNA was purified and used for whole-genome amplification as described previously ${ }^{14}$. The samples then underwent library preparation using the Illumina TruSeq Nano DNA Library Prep Kit following the manufacturer's instructions. Libraries were sequenced on an Illumina HiSeq 2500 sequencer to obtain $>10$ million uniquely mapped 50-bp single-end reads.

ChIP-Seq data was processed as described ${ }^{14}$. To generate the H3K27ac tracks shown in Fig. 3b, sequencing coverage was calculated for the six regions $(+/-1 \mathrm{~kb}$ around the SNP site of interest) at $50 \mathrm{bp}$ windows after extending each read to a length of $250 \mathrm{bp}$ along the sequencing direction using MEDIPS v.1.10.0 (extend $=250$, uniq $=F$, window_size $=50$, BSgenome $=$ 'BSgenome.Hsapiens.UCSC. $h 1^{\prime}$ ), and the resulting coverage profiles (normalized reads counts, RPKM) were presented. Further, to calculate fold change in H3K27ac enrichment levels between carriers of risk and non-risk allele at genomic regions harbouring the SNPs of interest (shown in Fig. 3c), we used the MEDIPS bioinformatics pipeline (as above) to obtain normalized reads counts for a 200 bp region around the SNP site (see Supplementary Data set 4). Genome-wide analysis of this entire data set will be reported in a separate manuscript (under preparation).

CTCF-ChIP and analysis of allele-specific binding. Purified chromatin from $10 \times 10^{6}$ polarized $\mathrm{T}_{\mathrm{H}} 1$ and $\mathrm{T}_{\mathrm{H}} 2$ cells ( 31 samples from 11 donors) and $1 \times 10^{6}$ primary $\mathrm{CD}^{+} \mathrm{T}$ cells (13 samples from 9 donors) were immunoprecipitated with a polyclonal anti-CTCF antibody (Lot \#2142232; 07-729; EMD Millipore), resulting in a total of 44 assays (including 13 technical duplicates). Chromatin was incubated with $10 \mu \mathrm{l}$ or $5 \mu \mathrm{l}$ antibody solution (for $10 \times 10^{6}$ cells or $1 \times 10^{6}$ cells ChIP, respectively), pre-coated with protein A-coated magnetic beads (Invitrogen). Immunocomplexes were captured, washed, and eluted and DNA was purified as described above. DNA sequences from control and target sites were quantified by real-time PCR (see Supplementary Table 1) to assess enrichment due to CTCF binding. Data are presented as fold enrichment over control region, which has previously been shown to not bind $\mathrm{CTCF}^{61}$. The donors were categorized (into homozygous risk, heterozygous and homozygous non-risk) based on allelic status of SNP overlapping the respective CTCF motif as shown in Fig. 4a and Supplementary Data set 3.

For allele-specific analysis, purified chromatin from input and CTCF-ChIP samples were amplified by PCR using primers that cover the CTCF-binding site overlapping SNPs rs12936231 (G/C; ZPBP2 site) and rs4065275 (A/G; ORMDL3 site), and analysed using the Sanger sequencing. Using MacVector, chromatogram traces of donors heterozygous for asthma risk SNPs (as above) were analysed to determine allele-preference for CTCF. The ratio of each allele was calculated using the signal intensity of each base at the position of the respective SNP. Data are shown as percentage of DNA sequences containing the risk and non-risk SNPs at rs12936231 (ZPBP2 site) or rs4065275 (ORMDL3 site).

Analyses of DHS and overlap with 17q21 SNPs. To define the collection of 17 q21 asthma-risk SNPs, we first downloaded all asthma-associated SNPs from the databases of GWAS Integrator ${ }^{62}$ and HaploReg v3 (ref. 63) (August 2015), yielding a total of 96 significant lead SNPs $\left(P<1.0 \times 10^{-5}\right)$ distributed over 75 loci (with 6 lead SNPs in the 17q21 locus). SNPs in tight genetic linkage $\left(r^{2}>0.8\right)$ were retrieved based on data from the Phase I of the 1,000 genome project using European (EUR) as reference population ${ }^{64}$ (calculations performed using HaploReg v3, Supplementary Data set 1). Among the total of 3,502 asthmaassociated SNPs (lead SNPs + linked SNPs), 136 SNPs were identified in the asthma-susceptibility locus on chromosome 17 (17q21).

To determine the number of DHS in the asthma loci, we downloaded genomic annotations of DHS from 64 primary cells types, 21 fetal tissues and 28 cell lines provided by the ENCODE Project Consortium (ENCODE Encyclopedia, version 2; http://www.encodeproject.org $)^{31}$ and the NIH Epigenomics Roadmap Consortium (http://www.roadmapepigenomics.org) ${ }^{32}$, and generated by the labs of Stamatoyannopoulos (University of Washington) and Crawford (Duke University).
We utilized processed data provided by the ENOCDE project; here the Stam lab merged all DNase peak data from the Stam and Crawford labs. This merging process formed one combined DNase-Seq data set with non-overlapping DHS. The Stam lab then identified the 'master' peak in each region, defined as the peak in the region with highest peak height/z-score. Utilizing this data set, loci were defined by the location of the respective SNPs extended for $10 \mathrm{~kb}$ at each end. We found a total of 462 unique DHS in the 17q21 asthma-risk locus (chr17: 37,899,254-38,139,253 (hg19); $240 \mathrm{~kb}$ ).

Next, to determine the average number of DHS in all loci for each cell type, we merged replicates from cell types of similar origin (see details in Supplementary Data set 2a). Of note, merged tracks of mixed or parental populations (for example data sets on 'CD3 cells' or 'CD4 primary cells') were excluded when data on subsets (such as 'CD4 naive cells', ' $\mathrm{T}_{\mathrm{H}} 1$ cells' and 'CD8 cells') were available. Our merged data set was used to determine the number of DHS within each cell type or tissue group (shown in Fig. 1d and Supplementary Fig. 1b) and to identify DHS that intersect directly with 17q21 SNPs (shown in Fig. 1e,f). The full list of merged DHS, including the number of single tracks for each cell type or tissue group, and the distribution of DHS in all asthma-associated loci (each defined by the location of the respective SNPs extended for $10 \mathrm{~kb}$ at each end) are provided in Supplementary Data set 2.

To predict the functional SNPs in the 17q21 locus, we performed motif scan analysis for the genomics regions highlighted in Fig. 3a using FIMO at the default parameters $\left(P<1 \times 10^{-4}\right)$, implementing the motif models from the JASPAR database $^{65}$ (see details Supplementary Data set 5 ).

4C-Seq assay and analysis. The 4C-Seq assay was used to determine regions interacting with the ORMDL3 promoter region ${ }^{40,41}$. Briefly, naive CD4 ${ }^{+} \mathrm{T}$ cells (or HUT-78 cells) were cross-linked and flash frozen. Cells were lysed, chromatin was digested using the 4-base cutter DpnII and restricted ends re-ligated in situ as described $^{36}$. The chromatin was de-crosslinked and digested using the 4 -base cutter MseI (or NlaIII) in order to produce small products ${ }^{40}$. Following a second ligation step in a large reaction volume (to minimize inter-molecular ligations), DNA sequences containing the ORMDL3 promoter region (anchor point/bait) were amplified by PCR (see Supplementary Table 1) and sequenced to generate 1-2 million $150 \mathrm{bp}$ single-end reads per sample. Conditions were initially optimized in HUT-78 cells using five different baits located in the ORMDL3 promoter region (baits A-E), and bait D, which showed most interactions, was chosen to perform the $4 \mathrm{C}$-Seq assay in primary $\mathrm{CD} 4{ }^{+} \mathrm{T}$ cells.

Raw fastq files were filtered to remove reads that do not contain the expected bait sequence. From the remaining reads, the bait sequence was removed and reads were mapped against the genomic sequence (hg19) using either Bowtie 1.1.2 $\left(4 \mathrm{C}-\mathrm{ker}^{42}\right)$ or the custom 4Cseqpipe ${ }^{40}$ mapper (perl 4cseqpipe.pl -map). Number of reads per each restriction enzyme fragment was computed using coverageBed (bedtools) on the fragmented genome (Supplementary Data set 7). Regions with significant interactions were calculated using 4C-ker (near-cis function with $K=5$ ). Differentially interacting regions at an adjusted $P$ value of 0.1 were called using the differential analysis function of the 4C-ker tool (Supplementary Data set 6). All domainograms (for example, Fig. 5c) were generated using the 4Cseqpipe tool (near-cis parameter; trend resolution of 5,000; using mean as the statistics).

Cell culture and stimulation. For siRNA knockdown studies memory CD4 ${ }^{+}$ T cells were cultured in Iscove's Modified Dulbecco's Medium (Invitrogen) supplemented with $5 \%(\mathrm{vol} / \mathrm{vol})$ heat-inactivated fetal bovine serum and $2 \%$ (vol/vol) human AB serum (CellGro) with recombinant human IL-7 (5 $\mathrm{ng} \mathrm{ml}^{-1}$. Miltenyi Biotec). For in vitro differentiation of naive cells into $\mathrm{T}_{\mathrm{H}} 1$ or $\mathrm{T}_{\mathrm{H}} 2$ cells, FACS-sorted naive CD4 ${ }^{+} \mathrm{T}$ cells were cultured with Human T-Activator CD3/CD28 Dynabeads (Invitrogen) at a bead-to-cell ratio of 1:1 in the presence of recombinant human IL-12 (rhIL-12; $5 \mathrm{ng} \mathrm{ml}^{-1}$ ) and anti-IL-4 antibody $\left(5 \mathrm{~g} \mathrm{ml}^{-1}\right)$, or recombinant human IL-4 (rhIL-4; $\left.10 \mathrm{ng} \mathrm{ml}^{-1}\right)$ and antiIFN- $\gamma$ antibody $\left(10 \mu \mathrm{g} \mathrm{ml}^{-1}\right)$, respectively. Recombinant IL-4, IL-12 and anti-IL-4 antibody were from R\&D Systems; the anti-IFN- $\gamma$ antibody was obtained from BD Pharmingen. After $48 \mathrm{~h}$ of culture, the anti-CD3/CD28 Dynabeads were removed and the cells were expanded in culture with recombinant human IL-2 (rhIL-2; $100 \mathrm{IU} \mathrm{ml}^{-1}$; National Cancer Institute). After 6 days, cells were washed and analysed for gene expression by real-time PCR. Memory CD4 ${ }^{+} \mathrm{T}$ cells were intracellularly stained for cytokine detection after stimulation with anti-CD3/CD28 Dynabeads for 6 and $18 \mathrm{~h}$. Brefeldin A $\left(5 \mu \mathrm{g} \mathrm{ml}^{-1}\right.$; Sigma-Aldrich) was added for the final $2 \mathrm{~h}$ of culture. The intracellular staining assay was performed as previously described $^{60}$. Data were acquired on a LSR-II (Becton Dickinson) and analysed using FlowJo software (Tree Star).

siRNA knockdown studies. Memory $\mathrm{CD} 4^{+} \mathrm{T}$ cells were transiently transfected with $0.5 \mathrm{nmol}$ (per $1 \times 10^{6}$ cells) of siRNA pools specific for ORMDL3, GSDMB, TBX21, and GATA3 or non-targeting siRNA (all ON-TARGETplus SMARTpools from Dharmacon) using the Neon Transfection System (Invitrogen) according to the manufacturer's protocol (settings: $2,200 \mathrm{~V}, 10 \mathrm{~ms}, 3$ pulses). All siRNA sequences are provided in Supplementary Table 3. Knockdown efficiency was analysed $48 \mathrm{~h}$ after transfection by real-time PCR for transcript levels, and by intracellular FACS assay for protein levels when specific and good antibodies were 
available for ORMDL (ab107639) as previously described ${ }^{45}$, T-bet (4B10) and GATA3 (L50-823) (Fig. 6b and Supplementary Fig. 6a,b); we also verified the effects of ORMDL3 and GSDMB siRNA pools by using the four independent siRNAs in the SMARTpool and the corresponding C911 mismatch control siRNAs ${ }^{66}$, where the nucleotide sequence is altered at bases 9-11, the seed sequence needed for specific targeting (Supplementary Fig. 6e,f and Supplementary Table 3). Viability of cells was analysed $48 \mathrm{~h}$ after transfection by using FACS-based Annexin-V and 4',6-diamidino-2-phenylindole (DAPI) staining. Subsequently, the memory $\mathrm{CD} 4{ }^{+} \mathrm{T}$ cells were stimulated with anti-CD3/CD28 Dynabeads and cytokine production was analysed by real-time PCR and intracellular FACS at various time points. Cytokine secretion after $48 \mathrm{~h}$ of stimulation was measured with V-PLEX Validated Assay Kits (Meso Scale Discovery) following the manufacturer's instructions. All data are presented as mean of biological duplicates.

Data availability. The authors declare that the data supporting the findings of this study are available within the paper (and its supplementary information files).

\section{References}

1. Moffatt, M. F. et al. Genetic variants regulating ORMDL3 expression contribute to the risk of childhood asthma. Nature 448, 470-U475 (2007).

2. Barrett, J. C. et al. Genome-wide association study and meta-analysis find that over 40 loci affect risk of type 1 diabetes. Nat. Genet. 41, 703-707 (2009).

3. Franke, A. et al. Genome-wide meta-analysis increases to 71 the number of confirmed Crohn's disease susceptibility loci. Nat. Genet. 42, 1118-1125 (2010).

4. McGovern, D. P. et al. Genome-wide association identifies multiple ulcerative colitis susceptibility loci. Nat. Genet. 42, 332-337 (2010).

5. Anderson, C. A. et al. Meta-analysis identifies 29 additional ulcerative colitis risk loci, increasing the number of confirmed associations to 47 . Nat. Genet. 43, 246-252 (2011)

6. Sheng, Y. et al. Sequencing-based approach identified three new susceptibility loci for psoriasis. Nat. Commun. 5, 4331 (2014)

7. Stahl, E. A. et al. Genome-wide association study meta-analysis identifies seven new rheumatoid arthritis risk loci. Nat. Genet. 42, 508-514 (2010).

8. Lessard, C. J. et al. Identification of IRF8, TMEM39A, and IKZF3-ZPBP2 as susceptibility loci for systemic lupus erythematosus in a large-scale multiracial replication study. Am. J. Hum. Genet. 90, 648-660 (2012).

9. Wellcome Trust Case Control Consortium. Genome-wide association study of 14,000 cases of seven common diseases and 3,000 shared controls. Nature 447, 661-678 (2007).

10. Visscher, P. M., Brown, M. A., McCarthy, M. I. \& Yang, J. Five years of GWAS discovery. Am. J. Hum. Genet. 90, 7-24 (2012).

11. Ernst, J. et al. Mapping and analysis of chromatin state dynamics in nine human cell types. Nature 473, 43-49 (2011).

12. Maurano, M. T. et al. Systematic localization of common disease-associated variation in regulatory DNA. Science 337, 1190-1195 (2012).

13. Gerasimova, A. et al. Predicting cell types and genetic variations contributing to disease by combining GWAS and epigenetic data. PLos ONE 8, e54359 (2013).

14. Seumois, G. et al. Epigenomic analysis of primary human T cells reveals enhancers associated with $\mathrm{T}(\mathrm{H}) 2$ memory cell differentiation and asthma susceptibility. Nat. Immunol. 15, 777 (2014).

15. Fang, Q., Zhao, H., Wang, A., Gong, Y. \& Liu, Q. Association of genetic variants in chromosome 17q21 and adult-onset asthma in a Chinese Han population. BMC Med. Genet. 12, 133 (2011).

16. Ferreira, M. A. R. et al. Association between ORMDL3, IL1RL1 and a deletion on chromosome 17q21 with asthma risk in Australia. Eur. J. Hum. Genet. 19, 458-464 (2011).

17. Flory, J. H. et al. 17q12-21 variants interact with smoke exposure as a risk factor for pediatric asthma but are equally associated with early-onset versus late-onset asthma in North Americans of European ancestry. J. Allergy. Clin. Immun. 124, 605-607 (2009).

18. Galanter, J. et al. ORMDL3 gene is associated with asthma in three ethnically diverse populations. Am. J. Resp. Crit. Care 177, 1194-1200 (2008).

19. Leung, T. F. et al. Asthma and atopy are associated with chromosome $17 \mathrm{q} 21$ markers in Chinese children. Allergy 64, 621-628 (2009).

20. Torgerson, D. G. et al. Meta-analysis of genome-wide association studies of asthma in ethnically diverse North American populations. Nat. Genet. 43, 887-892 (2011).

21. Wan, Y. I. et al. Genome-wide association study to identify genetic determinants of severe asthma. Thorax 67, 762-768 (2012).

22. Liu, X. et al. Genome-wide meta-analyses identify three loci associated with primary biliary cirrhosis. Nat. Genet. 42, 658-660 (2010).

23. Lluis, A. et al. Asthma-associated polymorphisms in $17 \mathrm{q} 21$ influence cord blood ORMDL3 and GSDMA gene expression and IL-17 secretion. J. Allergy Clin. Immunol. 127, 1587-1594 e1586 (2011).

24. Halapi, E. et al. A sequence variant on $17 \mathrm{q} 21$ is associated with age at onset and severity of asthma. Eur. J. Hum. Genet. 18, 902-908 (2010).
25. Murphy, A. et al. Mapping of numerous disease-associated expression polymorphisms in primary peripheral blood $\mathrm{CD}^{+}{ }^{+}$lymphocytes. Hum. Mol. Genet. 19, 4745-4757 (2010).

26. Caliskan, M. et al. Rhinovirus wheezing illness and genetic risk of childhood-onset asthma. New Engl. J. Med. 368, 1398-1407 (2013).

27. Verlaan, D. J. et al. Allele-specific chromatin remodeling in the ZPBP2/GSDMB/ORMDL3 locus associated with the risk of asthma and autoimmune disease. Am. J. Hum. Genet. 85, 377-393 (2009).

28. Locksley, R. M. Asthma and allergic inflammation. Cell 140, 777-783 (2010).

29. Scanlon, S. T. \& McKenzie, A. N. Type 2 innate lymphoid cells: new players in asthma and allergy. Curr. Opin. Immunol. 24, 707-712 (2012).

30. Erle, D. J. \& Sheppard, D. The cell biology of asthma. J. Cell Biol. 205, 621-631 (2014).

31. Encode Project Consortium. An integrated encyclopedia of DNA elements in the human genome. Nature 489, 57-74 (2012).

32. Bernstein, B. E. et al. The NIH Roadmap Epigenomics Mapping Consortium. Nat. Biotechnol. 28, 1045-1048 (2010).

33. Li, X. et al. eQTL of bronchial epithelial cells and bronchial alveolar lavage deciphers GWAS-identified asthma genes. Allergy 70, 1309-1318 (2015).

34. Kay, A. B. Allergy and allergic diseases. Second of two parts. New Engl. J. Med. 344, 109-113 (2001).

35. Shukla, S. et al. CTCF-promoted RNA polymerase II pausing links DNA methylation to splicing. Nature 479, 74-79 (2011).

36. Rao, S. S. et al. A 3D map of the human genome at kilobase resolution reveals principles of chromatin looping. Cell 159, 1665-1680 (2014).

37. Sanborn, A. L. et al. Chromatin extrusion explains key features of loop and domain formation in wild-type and engineered genomes. Proc. Natl Acad. Sci. USA 112, E6456-E6465 (2015).

38. Vietri Rudan, M. et al. Comparative Hi-C reveals that CTCF underlies evolution of chromosomal domain architecture. Cell Rep. 10, 1297-1309 (2015).

39. Ji, X. et al. 3D chromosome regulatory landscape of human pluripotent cells. Cell Stem Cell 18, 262-275 (2016).

40. van de Werken, H. J. et al. Robust 4C-seq data analysis to screen for regulatory DNA interactions. Nat. Methods 9, 969-972 (2012).

41. Stadhouders, R. et al. Multiplexed chromosome conformation capture sequencing for rapid genome-scale high-resolution detection of long-range chromatin interactions. Nat. Prot. 8, 509-524 (2013).

42. Raviram, R. et al. 4C-ker: A Method to Reproducibly Identify Genome-Wide Interactions Captured by 4C-Seq Experiments. PLoS Comput. Biol. 12, e1004780 (2016).

43. Cantero-Recasens, G., Fandos, C., Rubio-Moscardo, F., Valverde, M. A. \& Vicente, R. The asthma-associated ORMDL3 gene product regulates endoplasmic reticulum-mediated calcium signaling and cellular stress. Hum. Mol. Genet. 19, 111-121 (2010).

44. Siow, D. L. \& Wattenberg, B. W. Mammalian ORMDL proteins mediate the feedback response in ceramide biosynthesis. J. Biol. Chem. 287, 40198-40204 (2012).

45. Miller, M. et al. ORMDL3 is an inducible lung epithelial gene regulating metalloproteases, chemokines, OAS, and ATF6. Proc. Natl Acad. Sci. USA 109, 16648-16653 (2012).

46. Boyman, O. \& Sprent, J. The role of interleukin-2 during homeostasis and activation of the immune system. Nat. Rev. Immunol. 12, 180-190 (2012).

47. Moffatt, M. F. et al. A large-scale, consortium-based genomewide association study of asthma. New Engl. J. Med. 363, 1211-1221 (2010).

48. Breslow, D. K. et al. Orm family proteins mediate sphingolipid homeostasis. Nature 463, 1048-1053 (2010).

49. Han, S. M., Lone, M. A., Schneiter, R. \& Chang, A. Orm1 and Orm2 are conserved endoplasmic reticulum membrane proteins regulating lipid homeostasis and protein quality control. Proc. Natl Acad. Sci. USA 107, 5851-5856 (2010).

50. Carreras-Sureda, A. et al. ORMDL3 modulates store-operated calcium entry and lymphocyte activation. Hum. Mol. Genet. 22, 519-530 (2013).

51. Miller, M. et al. ORMDL3 transgenic mice have increased airway remodeling and airway responsiveness characteristic of asthma. J. Immunol. 192, 3475-3487 (2014).

52. Ha, S. G. et al. ORMDL3 promotes eosinophil trafficking and activation via regulation of integrins and CD48. Nat. Commun. 4, 2479 (2013).

53. Bugajev, V. et al. Negative regulatory roles of ORMDL3 in the FcepsilonRI-triggered expression of proinflammatory mediators and chemotactic response in murine mast cells. Cell. Mol. life Sci.: CMLS 73, 1265-1285 (2015).

54. Schedel, M. et al. Polymorphisms related to ORMDL3 are associated with asthma susceptibility, alterations in transcriptional regulation of ORMDL3, and changes in TH2 cytokine levels. J. Allergy Clin. Immunol. 136, 893-903 e814 (2015). 
55. Herold, M., Bartkuhn, M. \& Renkawitz, R. CTCF: insights into insulator function during development. Development 139, 1045-1057 (2012).

56. Kim, T. H. et al. Analysis of the vertebrate insulator protein CTCF-binding sites in the human genome. Cell 128, 1231-1245 (2007).

57. Ren, L. C. et al. CTCF mediates the cell-type specific spatial organization of the Kcnq5 locus and the local gene regulation. PLos ONE 7, e31416 (2012).

58. Tang, Z. et al. CTCF-mediated human 3D genome architecture reveals chromatin topology for transcription. Cell 163, 1611-1627 (2015).

59. Vijayanand, P. et al. Invariant natural killer $\mathrm{T}$ cells in asthma and chronic obstructive pulmonary disease. New Engl. J. Med. 356, 1410-1422 (2007).

60. Vijayanand, P. et al. Interleukin-4 production by follicular helper T cells requires the conserved Il4 enhancer hypersensitivity site V. Immunity 36, 175-187 (2012).

61. Wendt, K. S. et al. Cohesin mediates transcriptional insulation by CCCTCbinding factor. Nature 451, 796-801 (2008).

62. Yu, W. et al. GWAS Integrator: a bioinformatics tool to explore human genetic associations reported in published genome-wide association studies. Eur. J. Hum. Genet. 19, 1095-1099 (2011).

63. Ward, L. D. \& Kellis, M. HaploReg: a resource for exploring chromatin states, conservation, and regulatory motif alterations within sets of genetically linked variants. Nucleic Acids Res. 40, D930-D934 (2012).

64. Genomes Project Consortium et al. An integrated map of genetic variation from 1,092 human genomes. Nature 491, 56-65 (2012).

65. Grant, C. E., Bailey, T. L. \& Noble, W. S. FIMO: scanning for occurrences of a given motif. Bioinformatics 27, 1017-1018 (2011).

66. Buehler, E., Chen, Y. C. \& Martin, S. C911: A bench-level control for sequence specific siRNA off-target effects. PLos ONE 7, e51942 (2012).

\section{Acknowledgements}

We thank Cheryl Kim, Lara Nosworthy and Kurt van Gunst at the La Jolla Institute (LJI) flow cytometry facility for assisting with cell sorting; Alex Fu, Moghaddas Gholami and Jason Greenbaum at the LJI bioinformatics core for help with processing and analysis of sequencing data; the ENCODE Project and NIH Epigenomics Roadmap Consortiums, the laboratories of Stamatoyannopoulos (University of Washington), Crawford (Duke University) and Bernstein and Meissner (Broad Institute) for generating publically available data sets on DHS, H3K27ac and H3K4mel enrichment; and Nunzio Bottini,
Anjana Rao and Jessica Moore for careful reviewing of the manuscript. This work was funded by NIH grants R01 HL114093 and R24 AI108564 (P.V., B.P.), U19 AI100275 (P.V., B.P., A.S.) and The William K. Bowes Jr Foundation (P.V.).

\section{Author contributions}

B.J.S., A.S., B.P. and P.V. conceived the work, designed, performed and analysed experiments, and wrote the paper; D.S.-C. performed bioinformatic analyses under the supervision of B.J.S., L.C. and P.V.; F.A. supervised the analysis of 4C-Seq data and provided statistical input for the bioinformatics analysis; J.C. performed micro-scaled ChIP-Seq experiments under the supervision of G.S. and P.V.; V.S. performed T cell assays.

\section{Additional information}

Supplementary Information accompanies this paper at http://www.nature.com/ naturecommunications

Competing financial interests: The authors declare no competing financial interests.

Reprints and permission information is available online at http://npg.nature.com/ reprintsandpermissions/

How to cite this article: Schmiedel, B. J. et al. 17q21 asthma-risk variants switch CTCF binding and regulate IL-2 production by T cells. Nat. Commun. 7, 13426 doi: 10.1038/ ncomms13426 (2016).

Publisher's note: Springer Nature remains neutral with regard to jurisdictional claims in published maps and institutional affiliations.

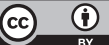

This work is licensed under a Creative Commons Attribution 4.0 International License. The images or other third party material in this article are included in the article's Creative Commons license, unless indicated otherwise in the credit line; if the material is not included under the Creative Commons license, users will need to obtain permission from the license holder to reproduce the material. To view a copy of this license, visit http://creativecommons.org/licenses/by/4.0/

(C) The Author(s) 2016 